\title{
MicroRNA-1929-3p participates in murine cytomegalovirus-induced hypertensive vascular remodeling through Ednra/NLRP3 inflammasome activation
}

\author{
WEI ZHOU ${ }^{1 *}$, DONGMEI XI ${ }^{1 *}$, YUNZHONG SHI $^{1 *}$, LAMEI WANG $^{2}$, HUA ZHONG $^{1}$, \\ ZHEN HUANG ${ }^{1}$, YONGMIN LIU ${ }^{1}$, YAN TANG ${ }^{3}$, NING LU ${ }^{1}$, YONGJIA WANG ${ }^{1}$, \\ ZHENGYU ZHANG ${ }^{4}$, JIAXIN PEI ${ }^{4}$, NA TANG ${ }^{1}$ and FANG HE ${ }^{1}$
}

\begin{abstract}
${ }^{1}$ Department of Pathophysiology, Key Laboratory of Education Ministry of Xinjiang Endemic and Ethnic Diseases, Medical College of Shihezi University; ${ }^{2}$ Centre of Medical Functional Experiments, Medical College of Shihezi University;

${ }^{3}$ Department of Geriatrics, The First Affiliated Hospital of Medical College of Shihezi University; ${ }^{4}$ Department of Clinical Medicine, Medical College of Shihezi University, Shihezi, Xinjiang 832002, P.R. China
\end{abstract}

Received August 25, 2020; Accepted November 16, 2020

DOI: $10.3892 / \mathrm{ijmm} .2020 .4829$

\begin{abstract}
MicroRNAs (miRNAs) play an important role in the development of vascular remodeling in essential hypertension (EH) by mediating the effects of human cytomegalovirus (HCMV) on the vascular system. Therefore, the aim of the present study was to investigate the effects of murine cytomegalovirus (MCMV) infection on blood pressure and vascular function in mice, in order to elucidate the role of miR-1929-3p in this process. For model development, 7-month-old C57BL/6J mice were infected with the Smith strain of MCMV, and MCMV DNA, IgG and IgM were detected. Subsequently, blood pressure was measured via the carotid artery, and the morphological changes of the aorta were evaluated by hematoxylin and eosin and Masson's trichrome staining. miR-1929-3p transfection was performed using an adeno-associated virus packaged vector and the changes in vascular structure were then observed. The levels of nitric oxide (NO) and endothelial NO synthase were also assessed with colorimetry. Vascular remodeling and expression of NLRP3 inflammasome pathway-related proteins were detected by immunohistochemistry and western blotting. Endothelin-1 (ET-1), interleukin (IL)-1 $\beta$ and IL-18 were assayed by ELISA. The results revealed that MCMV infection increased the blood pressure, promoted vascular
\end{abstract}

Correspondence to: Dr Na Tang or Dr Fang He, Department of Pathophysiology, Key Laboratory of Education Ministry of Xinjiang Endemic and Ethnic Diseases, Medical College of Shihezi University, 59 North 2nd Road, Shihezi, Xinjiang 832002, P.R. China E-mail: tangna2014ssry@163.com

E-mail: fangf2002shz@126.com

*Contributed equally

Key words: essential hypertension, murine cytomegalovirus, vascular remodeling, miR-1929-3p, Ednra, NLRP3 remodeling, caused endothelial cell injury, and downregulated miR-1929-3p. However, these effects were alleviated by miR-1929-3p overexpression, which downregulated endothelin A receptor (Ednra) and NLRP3 inflammasome, as well as endothelial injury- and vascular remodeling-related genes. Taken together, the findings of the present study indicated that overexpression of miR-1929-3p may improve MCMV-induced vascular remodeling, possibly via the deactivation of the NLRP3 inflammasome by ET-1/Ednra.

\section{Introduction}

Hypertension is a frequently occurring disease causing cardiovascular complications (1), which currently represents one of the most serious public health concerns and a major socioeconomic burden. Vascular remodeling is a critical pathological characteristic of hypertension, leading to increased vascular resistance and hemodynamic changes (1). Approximately 95\% of the cases are classed as essential hypertension (EH) (2-4); however, to date, the exact etiology and pathogenesis of EH have not been fully elucidated. Recent studies have found that the pathogenic factors of EH do not only involve the interaction between genetic and environmental factors $(5,6)$, but are also correlated with human cytomegalovirus (HCMV) infection, microRNA (miRNA) regulation and inflammation activation, as well as other mechanisms (7-9).

HCMV is a ubiquitous beta-herpes virus with the largest genome among human herpesviruses, the gene products of which play immunomodulatory roles in the host $(10,11)$. The findings of epidemiological studies indicate that the prevalence of HCMV in the population is $40-100 \%$, depending on socioeconomic and geographical factors (12). CMV is reportedly associated with $\mathrm{EH}(13,14)$. When HCMV infects humans, it may enter a latent infection phase and cause disease by changing host and viral miRNA expression (15). miRNAs are single-stranded, non-coding RNAs composed of 20-25 nucleotides, which can inhibit protein synthesis by inhibiting the expression of specific target genes or directly terminating the translation of target 
mRNAs $(16,17)$. Furthermore, accumulating evidence suggests that miR-21 plays an important role in vascular remodeling in hypertension (18). Additionally, the deletion of miR-431-5p can prevent hypertension and vascular injury caused by angiotensin II (Ang II) (19). However, decreased expression of miR-181-5p participates in the phenotypic transformation of the vascular smooth muscle cells (VSMCs) in Ang II-induced hypertension by causing an increase in high mobility group box 1 (20). It was previously confirmed that HCMV-encoded miR-UL112 promoted HCMV-mediated vascular disease by inducing vascular endothelial cell dysfunction (21). It was also reported that miR-138 promoted the migration of human umbilical vein endothelial cells (HUVECs) and tube formation in HCMV-infected HUVECs via the SIRT1/P-STAT3 pathway (22). However, the virus encodes at least 26 mature miRNAs (23-25). Given the regulatory network of miRNAs and their important role in the pathogenesis of hypertension, the recently discovered HCMV-encoded miRNAs have not sufficiently elucidated the role and underlying mechanism of action of CMV and associated miRNAs in hypertension. It remains unclear whether miRNA changes in the host following CMV infection are involved in the occurrence and development of hypertension and vascular remodeling.

The nucleotide-binding domain, leucine-rich repeat protein 3 (NLRP3) inflammasome, is a multiprotein complex consisting of an NLRP3 scaffold, an adaptor apoptosis speck-like protein containing a CARD (ASC) and the effector procaspase-1, and participates in the activation of the inflammatory response. Activation and assembly of inflammasomes promote proteolytic cleavage, maturation and secretion of the pro-inflammatory cytokines interleukin (IL)-1ß and IL-18 (26). Accumulating evidence suggests that $\mathrm{EH}$ is also associated with NLRP3 inflammasome activation $(27,28)$ and, in turn, the inflammatory response is involved in end-organ injury and vascular remodeling in EH (29). Moreover, NLRP3 is closely associated with certain miRNAs, and miR-181a has been demonstrated to activate the NLRP3 inflammatory pathway by targeting mitogen-activated protein kinase kinase 1 in atherosclerosis (30). Moreover, miR-125a-5p negatively regulates the NLRP3 inflammasome by targeting C-C motif chemokine ligand 4 in human VSMCs treated with oxidized low-density lipoprotein (31). NLRP3 inflammasome activation also plays an important role in the phenotypic transformation and proliferation of human VSMCs and vascular remodeling $(32,33)$.

We previously observed that blood pressure was increased and miR-1929-3p was significantly downregulated in mouse cytomegalovirus (MCMV)-infected-C57BL/6J mice (34). Moreover, using bioinformatics analysis and the dual-luciferase reported assay, the endothelin A receptor (Ednra) was validated as one of the hypertension-associated target genes regulated by miR-1929-3p. It has been demonstrated that endothelin-1 (ET-1) induces vascular remodeling-related persistent inflammation through Ednra (35). Based on our previous experimental results and the aforementioned associations among MCMV, miRNAs and the NLRP3 inflammasome, it was hypothesized that MCMV infection may decrease the expression of miR-1929-3p in mice, thereby relieving the inhibition of Ednra and activating the NLRP3 inflammasome in order to promote the occurrence and development of adverse vascular remodeling and hypertension.

\section{Materials and methods}

Animals. All experimental procedures involving laboratory animals were approved by the Institutional Animal Research Committee at Shihezi University Medical College. A total of 200 C57BL/6J male mice, aged 7 months and weighing 25-30 g, were purchased from Beijing Viton Lihua Laboratory Animal Co., Ltd. (license no. SCXK 2016-0006). During the whole study period, the animals were housed at room temperature with 12-h light/dark cycles and allowed access to normal rat chow and water ad libitum. The mice were randomly divided into groups ( $\mathrm{n}=17 /$ group) as follows: Control, MCMV, MCMV + miR-NC and MCMV + miR-1929-3p. In the MCMV group, mice were intraperitoneally injected with $5 \times 10^{4} \mathrm{PFU} / \mathrm{animal}$, while the control group was inoculated with the same amount of normal saline. In the miR-1929-3p and miR-1929-3p negative control (miR-NC) groups, mice were injected via the tail vein with rAAV-miR-1929-3p or rAAV-miR-1929-3p-NC (1×10 11 virion particles in $100 \mathrm{ml}$ saline solution). The mice were anesthetized using sodium pentobarbital (P3761, Sigma-Aldrich; Merck $\mathrm{KGaA})$ through intraperitoneal injection $(30 \mathrm{mg} / \mathrm{kg}$ ), and blood was collected from the inner canthus artery. The mice were sacrificed by cervical dislocation and the aorta was resected, fixed in $4 \%$ paraformaldehyde at $4^{\circ} \mathrm{C}$ for $24 \mathrm{~h}$ and embedded in paraffin.

MCMV immediate early (IE) gene detection. Samples of mouse aorta $(30 \mathrm{mg})$ were homogenized into a tissue suspension, and the tissue DNA was extracted and amplified using a DNA extraction kit (Tiangen Biotech Co., Ltd.) using the following primers: MCMV IE forward, 5'-ATGGTGAAG CTATCAAAGATGTGCATCTCA-3' and reverse, 5'-ATC AATCAGCCATCAACTCTGCTACCACAC-3'. After agarose gel electrophoresis, the DNA was visualized on the Image-Pro Plus software, version 6.0 (Media Cybernetics, Inc.).

Blood pressure measurement. The C57BL/6 mice were anesthetized using sodium pentobarbital (P3761; Sigma-Aldrich; Merck KGaA) through intraperitoneal injection $(30 \mathrm{mg} / \mathrm{kg})$, after which time they were fixed on the operating table. An incision was made along the midline of the neck, and the neck tissues were carefully separated to expose the common carotid artery without injuring the vagus nerve. Next, a cannula was inserted in the artery and secured in place with $6 / 0$ silk threads, and the common carotid artery was repositioned. Systolic blood pressure (SBP), diastolic blood pressure (DBP) and mean arterial pressure (MAP) in the artery were monitored. For each measurement, the data represented the mean of at least 5 stable recordings.

Hematoxylin and eosin $(H \& E)$ and Masson's trichrome staining. The blood vessels were placed in $4 \%$ paraformaldehyde buffer $(\mathrm{pH}$ 7.0) and fixed at room temperature for $24 \mathrm{~h}$. The tissues were embedded in paraffin, cut into 4- $\mu \mathrm{m}$ sections, stained with H\&E (Solarbio) and Masson's trichrome stain (Beijing Solarbio Science \& Technology Co., Ltd.), and observed under a light microscopy (IX73, Olympus Corporation). Five microscopic fields (magnification, $\mathrm{x} 400$ ) were randomly selected from each blood vessel, and the collagen area was calculated as a percentage of the field of view. 
Colorimetry. After homogenizing the mouse blood vessel tissue, the supernatant was collected and analyzed for nitric oxide (NO) and endothelial NO synthase (eNOS) levels in mouse aortic homogenates using a commercial kit (Nanjing Jiancheng Biological Engineering Institute), according to the manufacturer's instructions.

Western blotting. After treatment, all experimental samples were collected and homogenized on ice in RIPA buffer containing $1 \%$ proteinase inhibitor solution. Then, the homogenates were centrifuged at $12,000 \mathrm{x} \mathrm{g}$ for $10 \mathrm{~min}$ at $4^{\circ} \mathrm{C}$. The resulting supernatant contained the extracted proteins. The concentration of total extracted protein was determined using NanoDrop2000 (Thermo Fisher Scientific, Inc.). A total of $10 \mu \mathrm{g}$ protein per lane were separated by electrophoresis on $10 \%$ SDS-PAGE gels before transferring onto a PVDF membrane by SEMI-DRY TRANSFER CELL (Bio-Rad Laboratories, Inc.). After blocking with 5\% non-fat dry milk for $3 \mathrm{~h}$ at room temperature, the membranes were incubated overnight at $4{ }^{\circ} \mathrm{C}$ with primary antibodies diluted in a $5 \%$ bovine serum albumin (NeoFROXX $\mathrm{GmbH}$ ) solution in $1 \mathrm{X}$ Tris-buffered saline solution containing $0.1 \%$ Tween-20 (TBS-T). The antibodies used for western blot analysis were as follows: $\beta$-actin $(1: 1,000$, cat. no. ZM-0001; Sugisuke Bridge), $\alpha$-smooth muscle actin ( $\alpha$-SMA; 1:400, cat. no. BM0002; Boster Biological Technology), osteopontin (OPN; 1:200, cat. no. ab8448; Abcam), proliferating cell nuclear antigen (PCNA; 1:250, cat. no. BM0104; Boster Biological Technology), NLRP3 (1:500, cat. no. ab214185; Abcam) ASC (1:250, cat. no. ab47092; Abcam), caspase-1 (1:1,000, cat. no. ab1872; Abcam), pro-caspase-1 (1:1,000, cat. no. ab179515; Abcam), IL-18 (1:1,000, cat. no. ab71495; Abcam), IL-1 $\beta$ (1:1,000, cat. no. ab9722; Abcam) and pro-IL-1 $\beta$ (1:1,000, cat. no. ab216995; Abcam). Subsequently, the membranes were washed three times with TBS-T and treated with goat anti-rabbit $\operatorname{lgG}(1: 10,000$; cat. no. ZB2301; Sugisuke Bridge, Beijing, China) or goat anti-rat $\lg G$ (1:10,000; cat. no. ZB2305; Sugisuke Bridge, Beijing, China) for $2 \mathrm{~h}$ at room temperature. The signals were visualized with Supersensitive ECL Chemiluminescent Kit, according to the manufacturer's instructions. The films w ere scanned and analyzed using the Image-Pro Plus software, version 6.0 (Media Cybernetics, Inc.).

$R N A$ extraction and reverse transcription-quantitative $P C R$ $(R T-q P C R)$ analysis. Total RNA was extracted from the collected aortic samples using TRIzol ${ }^{\circledR}$ (Invitrogen; Thermo Fisher Scientific, Inc.), treated with DNase I (Tiangen Biotech Co., Ltd.), reverse transcribed with SuperScript ${ }^{\mathrm{TM}}$ First Strand cDNA System (Invitrogen; Thermo Fisher Scientific, Inc.), and mature miRNA levels were assessed using SYBR Green Real-time PCR (Tiangen Biotech Co., Ltd.) according to the manufacturer's instructions with U6 and GAPDH as the reference genes. The thermocycling conditions were as follows: 3 min at $95^{\circ} \mathrm{C}$ followed by 40 cycles of amplification at $94^{\circ} \mathrm{C}$ for $20 \mathrm{sec}$ and at $60^{\circ} \mathrm{C}$ for $34 \mathrm{sec}$. The results were analyzed using the $2^{-\Delta \Delta C q}$ method (36). The sequences of the PCR primers used were as follows: miR-1929-3p forward, 5'-ACACTCCAGCTG GGCAGCTCATGGAGACCT-3' and reverse, 5'-TGGTGTCGT GGAGTCG-3'; Ednra forward, 5'-TCACCGTCTTGAACCTCT
GTGC-3' and reverse, 5'-GATGGAGACGATTTCAATGGC GG-3'; U6 forward, 5'-GCTTCGGCAGCACATATACTAAAA T-3' and reverse, 5'-CGCTTCACGAATTTGCGTGTCAT-3'; and GAPDH forward, 5'-TGGCCTTCCGTGTTCCTAC-3' and reverse, 5'-GAGTTGCTGTTGAAGTCGCA-3'.

Immunohistochemistry. To determine the expression of target proteins in the aortic tissues, the samples were stained by immunohistochemistry. Briefly, the aortic tissues were fixed in $4 \%$ paraformaldehyde for $24 \mathrm{~h}$ at $4^{\circ} \mathrm{C}$ and embedded in paraffin. Then, samples were cut into $3-\mu \mathrm{m}$ sections, rehydrated with $3 \% \mathrm{H}_{2} \mathrm{O}_{2}$ after being deparaffinized, and washed with $0.1 \mathrm{M}$ PBS. The sections were then incubated with primary antibodies at $4^{\circ} \mathrm{C}$ overnight. The antibodies used were as follows: OPN (1:100, cat. no. ab8448; Abcam), PCNA (1:50, cat. no. ab92552; Abcam), $\alpha$-SMA (1:200, cat. no. ab5694; Abcam), NLRP3 (1:100, cat. no. ab214185; Abcam), ASC (1:100, cat. no. ab47092; Abcam) and caspase-1 (1:25, cat. no. ab1872; Abcam). After washing with PBS, the sections were incubated with secondary antibody (Invitrogen; Thermo Fisher Scientific, Inc.) at room temperature for $30 \mathrm{~min}$. Then, color in the aorta was developed using 3,3-diaminobenzidine. The sections were then counterstained with hematoxylin at room temperature for $4 \mathrm{~min}$, incubated with ammonia for 20 min, dehydrated with an ethanol gradient (80\% ethanol for $5 \mathrm{~min}$; $95 \%$ ethanol for $5 \mathrm{~min}$; and $100 \%$ ethanol for $5 \mathrm{~min}$ ) and transparentized twice with xylene ( 5 min each time), mounted on glass coverslips, and sealed with neutral resin. Cells containing brown-strained particles in the cytoplasm were considered as positive based on imaging under a fluorescence microscope (Carl Zeiss AG). Quantitative image analyses were performed with the Image-Pro Plus software, version 6.0 (Media Cybernetics, Inc.). The mean of the number of positive cells from at least 5 random fields was calculated for each section and then averaged within the group. The staining scores were based on percentage of cells stained and intensity of staining: The level of protein accumulation was scored as 0 (no detectable immunostaining), 1 (few scattered stained nuclei), 2 (up to $10 \%$ stained nuclei), 3 (10-50\% stained nuclei), and 4 (>50\% stained nuclei) (37).

ELISA. Commercially available enzyme immunoassay kits (Jingmei Biotechnology) were used to detect MCMV IgG (cat. no. JM-02340M1), MCMV IgM (cat. no. JM-02341M1), IL-18 (cat. no. JM-02452M1), IL-1 $\beta$ (cat. no. JM-02323M1) and ET-1 (cat. no. JM-02844M1) levels in the plasma or aortic tissues. A microplate reader (Model 3550-UV; Bio-Rad Laboratories, Inc.) was used to measure the absorbance at a wavelength of $450 \mathrm{~nm}$.

Statistical analysis. GraphPad Prism 5.0 (GraphPad Software, Inc.) was used for statistical analyses. All the data are presented as mean \pm SD from at least three independent experiments, and all in vitro experiments were conducted in triplicate. The SPSS 20.0 statistical software (IBM Corp.) was utilized for statistical analysis. The Student's t-test was used for comparisons between two groups. Multiple comparisons were performed using one-way or two-way ANOVA. Tukey's multiple comparisons test was used for the pairwise comparison following ANOVA. $\mathrm{P}<0.05$ was considered to indicate a statistically significant difference. 


\section{Results}

Development of MCMV-infected C57BL/6J mouse model. In order to establish an experimental animal model for elevated blood pressure induced by MCMV infection, 7-month-old C57BL/6J mice were intraperitoneally injected with the Smith strain of MCMV, whereas control group mice were injected with the same amount of normal saline. At 9 months of age, the serum concentrations of MCMV IgG and $\operatorname{IgM}$ in the MCMV group were significantly higher compared with those in control group mice of the same age (Fig. 1A and B). PCR analysis revealed that the expression of MCMV IE in the MCMV group was positive, indicating that the mice had been infected with MCMV (Fig. 1C).

MCMV infection increases blood pressure and induces vascular remodeling in C57BL/6J mice. To determine the role of MCMV infection in mice with hypertension, blood pressure in mice was determined by carotid arterial pressure measurement. The results revealed that SBP, DBP and MAP in the MCMV group of 9- and 12-month mice were significantly higher compared with those in control group mice of the same age (Fig. 2A and C). This indicated that MCMV infection promoted an increase in blood pressure in mice. Concurrently, the miR-1929-3p expression level in the MCMV group decreased significantly with the increase in blood pressure (Fig. 2D). H\&E and Masson's trichrome staining revealed increased vascular media thickness and collagen accumulation in aortic tissue sections of 12-month-old mice in the MCMV group (Fig. 2E-H). Taken together, these results indicated that MCMV infection caused increased blood pressure and vascular remodeling in $\mathrm{C} 57 \mathrm{BL} / 6 \mathrm{~J}$ mice.

Overexpression of miR-1929-3p improves vascular remodeling in MCMV-induced hypertensive mice. To investigate the role of miR-1929-3p in vascular remodeling, the type 9 recombinant adeno-associated virus (rAAV9) system was used in MCMV-induced hypertensive C57BL/6J mice. At 13 and 15 months of age, the expression of miR-1929-3p in the MCMV group was significantly downregulated, while in the MCMV + miR-1929-3p group, miR-1929-3p expression was significantly increased (Fig. 3A). At the age of 14 months, blood pressure in MCMV + miR-1929-3p group mice was restored. This indicated that the overexpression of miR-1929-3p attenuated the MCMV-induced elevation of DBP, MAP and SBP (Fig. 3B-D).

A characteristic change caused by the increase in blood pressure is vascular remodeling due to the proliferation of VSMCs (4), with the morphological changes mainly comprising thickening of the media, increased rigidity of the vessel wall, and decreased vascular compliance (13). In the present study, the MCMV + miR-1929-3p group mice exhibited no significant changes at 13 months of age compared with the MCMV group. However, H\&E and Masson's trichrome staining of aortic tissue in mice aged 15 months revealed that miR-1929-3p significantly reduced the thickness of the aortic media and the fibrotic area of aortic tissue, which were caused by MCMV infection (Fig. 3E-H).

To further determine the role of miR-1929-3p in vascular remodeling in $\mathrm{MCMV}$-infected aortic tissues, the protein expression of $\alpha$-SMA, OPN and PCNA was analyzed using immunohistochemistry and western blotting. The results revealed that, at 15 months of age, the miR-1929-3p overexpression group exhibited an increase in the contractile phenotype of $\alpha$-SMA expression and a decrease in the synthetic phenotype of OPN and PCNA expression compared with the MCMV group (Fig. 4A-J). These data indicated that miR-1929-3p overexpression inhibited vascular smooth muscle thickening and exerted a protective effect against vascular remodeling. These findings indicated that MCMV infection promoted aortic remodeling by inhibiting the expression of miR-1929-3p, thereby leading to hypertension.

Targeted regulation of endothelin A receptor by miR-1929-3p alleviates endothelial dysfunction induced by MCMV infection. Ednra was previously identified as the target of miR-1929-3p (34). To confirm this finding, the expression level of Ednra was detected after overexpression of miR-1929-3p and, consistently with the results of the previous study, Ednra mRNA and protein expressions were upregulated in the MCMV group compared with those in the control group. However, Ednra mRNA and protein expression levels were significantly downregulated in the MCMV + miR-1929-3p group (Fig. 5B-D). These results indicated that MCMV infection downregulated miR-1929-3p and increased the expression of its target gene, Ednra, while miR-1929-3p overexpression alleviated the MCMV infection-induced increase of Ednra expression. It has been reported that the overexpression of ET-1 causes persistently elevated blood pressure and vascular injury through Ednra (38). Moreover, it has been demonstrated that endothelial cell dysfunction is an important component of inflammation caused by CMV infection (39). To assess the role of miR-1929-3p in MCMV-induced endothelial dysfunction, eNOS activity and NO content were measured as indicators of endothelial cell function in aortic tissue, and the content of ET-1 in plasma was also measured. Compared with the control group, plasma ET-1 concentration in mice in the MCMV group was significantly increased, while eNOS activity in aortic tissues was downregulated. However, plasma ET-1 concentration and aortic eNOS activity were significantly decreased in the MCMV group relative to the MCMV + miR-NC group after miR-1929-3p overexpression, up to 15 months of age. Additionally, the concentration of NO in the aortic tissues displayed the same trend as that of the activity of eNOS $(\mathrm{P}<0.05$; Fig. 5E-G).

These data suggested that MCMV infection may lead to endothelial cell dysfunction by inhibiting miR-1929-3p, increasing Ednra expression and promoting the production of endothelial injury factors, whereas overexpression of miR-1929-3p protects against MCMV-induced endothelial function disruption.

miR-1929-3p is involved in vascular remodeling associated with MCMV infection by activating the NLRP3 inflammasome. NLRP3 inflammasome activation reportedly triggers an inflammatory response and promotes vascular remodeling (8). Furthermore, it has been reported that ET-1-activated Ednra promotes the activation of the NLRP3 inflammasome (40). To explore the role of the NLRP3 inflammasome during the overexpression of miR-1929-3p in improving MCMV-induced 
$\mathbf{A}$

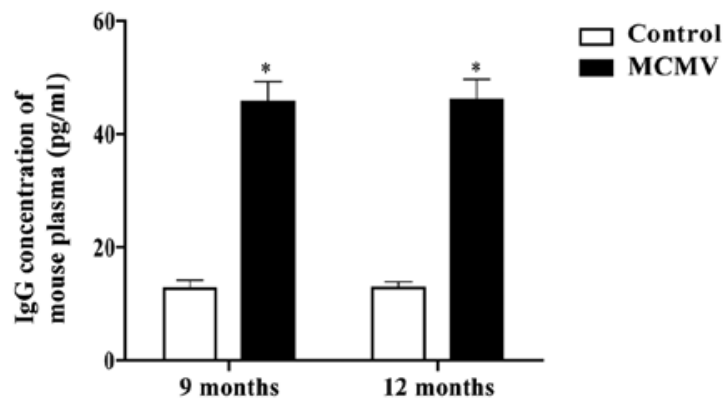

C

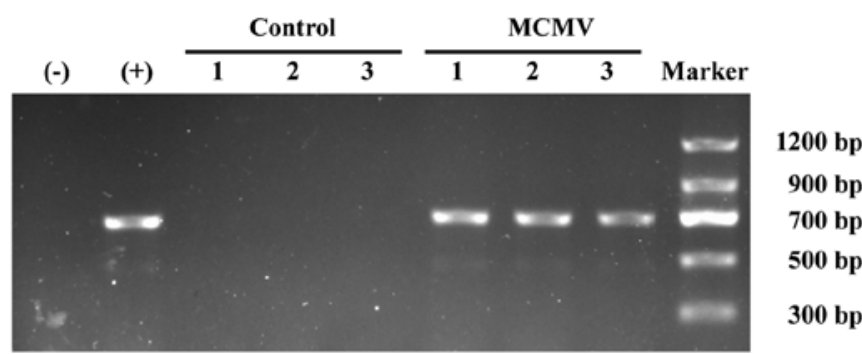

B

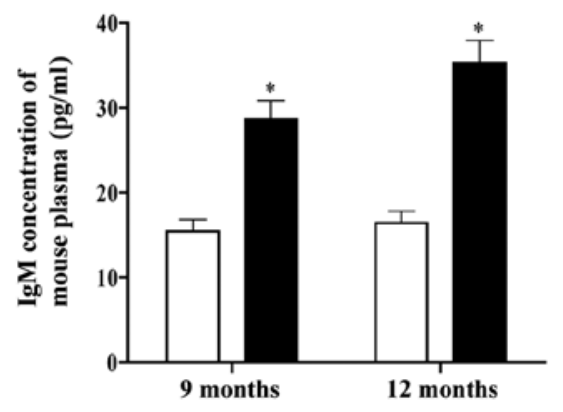

Figure 1. Development of MCMV-infected C57BL/6J mouse model. (A and B) The concentrations of IgG and IgM in the plasma were detected by ELISA. (C) PCR identified MCMV immediate early DNA in the aorta of C57BL/6J mice infected with MCMV. * $\mathrm{P}<0.05$ vs. control; in panels A and B, data were analyzed using the Student's t-test. $\mathrm{n}=5$ per group. MCMV, murine cytomegalovirus.

hypertension vascular remodeling, the expression of the NLRP3 inflammasome in mouse blood vessels was evaluated.

Immunohistochemical staining revealed that the levels of NLRP3, ASC and caspase-1 were markedly upregulated in the aortic tissues in the MCMV group at 13 and 15 months of age. NLRP3, ASC and caspase-1 expression levels did not change significantly in the MCMV + miR-1929-3p group at the age of 13 months, but were significantly downregulated at 15 months (Fig. 6A-F). The western blot analysis revealed that the expression of the NLRP3 inflammasome-related proteins, NLRP3, ASC, pro-caspase-1, caspase-1, pro-IL-1 and IL-1 $\beta$ were significantly increased in the MCMV-infected group. MCMV activated caspase-1 and IL-1 $\beta$; however, no significant difference in IL-18 expression was observed. These upregulated indicators were alleviated by miR-1929-3p overexpression in 15-month-old mice (Fig. 7A-J). Similarly, ELISA detected IL-18 and IL-1 $\beta$ in the plasma, and the results of this assay corresponded to those of the western blot analysis (Fig. 7K and L). These data demonstrated that miR-1929-3p overexpression can improve MCMV-induced vascular remodeling, possibly by inhibiting the activation of the NLRP3 inflammasome via ET-1/Ednra.

\section{Discussion}

To the best of our knowledge, the present study is the first to demonstrate that miR-1929-3p plays an important role in mediating hypertensive vascular remodeling in MCMV infection. It was observed that MCMV-infected mice had elevated blood pressure, thickened blood vessels, and decreased miR-1929-3p expression. When miR-1929-3p was overexpressed, the expression of its target gene, Ednra, decreased, while the activation of the NLRP3 inflammasome was inhibited, which improved
MCMV-induced vascular remodeling. In other words, miR-1929-3p overexpression may suppress MCMV-induced hypertensive vascular remodeling, indicating its potential value as a therapeutic target for hypertension. To the best of our knowledge, this is the first attempt at targeting miR-1929-3p for MCMV-induced hypertension therapeutics.

MCMV is widely invasive and can infect a variety of cells, including endothelial and smooth muscle cells (41). The fact that CMV infection may lead to an increase in blood pressure has also been observed in human studies (42). Hypertension is a common chronic disease, which is associated with age (43). Therefore, 7-month-old C57BL/6J mice infected MCMV were used to construct an animal model of hypertension (44). Blood pressure and vascular morphology detection were performed monthly. It was observed that SBP, DBP and MAP were significantly increased following MCMV infection at 9 months of age, while vascular morphology did not change significantly, suggesting that the blood pressure increase caused by MCMV infection occurred before the morphological changes of the blood vessels. Vascular remodeling is a characteristic phenotype in hypertension (45). This morphological change is mainly manifested by the thickening of the media, increased wall rigidity and decreased vascular compliance (46). It was observed that typical vascular remodeling, such as increased aortic thickness and collagen deposition in the aorta, occurred at 12 months of age in the MCMV group. The markers are shown primarily at 9 months of age, when blood pressure began to change, and at 12 months of age, when blood vessel function began to change. In addition, it was observed that blood pressure increases following MCMV infection precede changes in vascular function. This may be partly due to the fact that CMV re-infection usually presents with high levels of antibody titers and is involved in the development of 


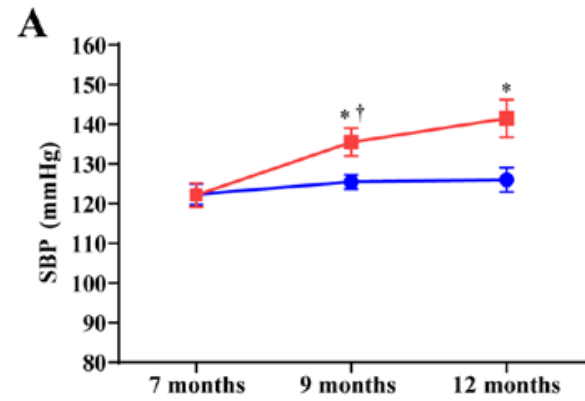

C

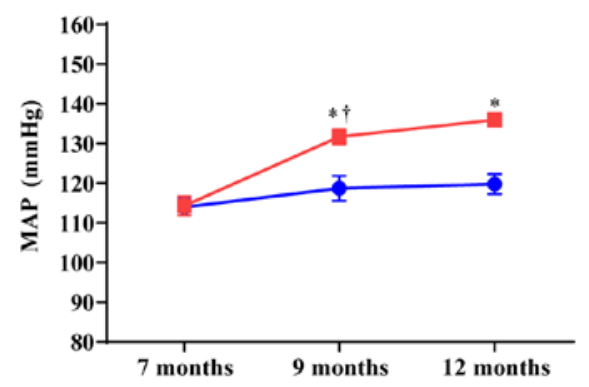

$\mathbf{E}$

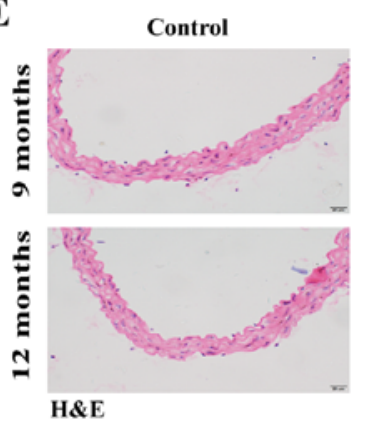

G

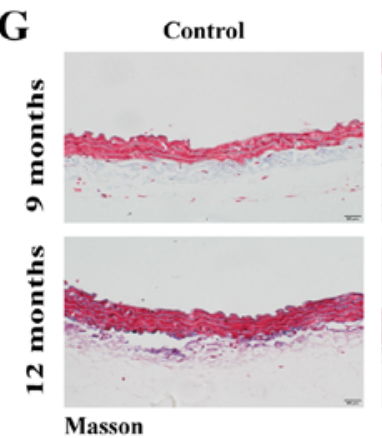

MCMV

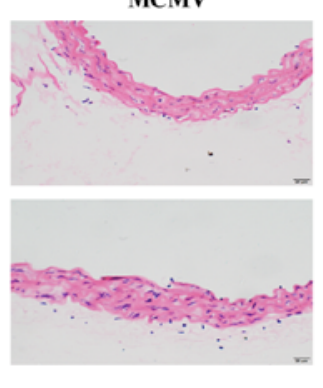

MCMV

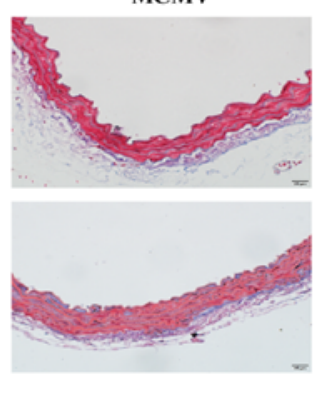

B

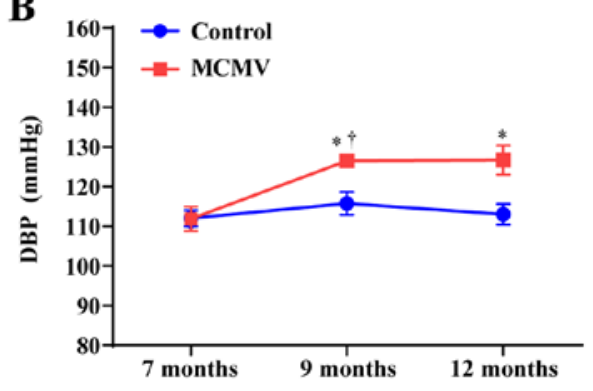

D

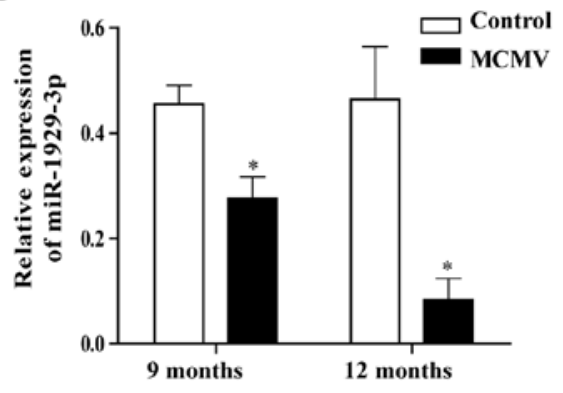

F

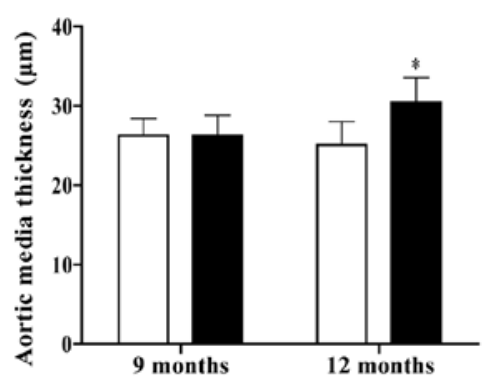

H

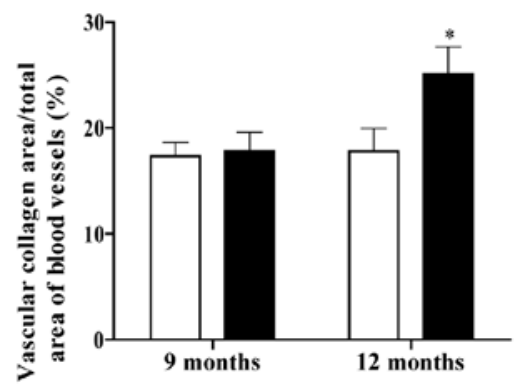

Figure 2. Increased blood pressure and vascular remodeling were caused by MCMV infection in C57BL/6J mice. (A) SBP, (B) DBP and (C) MAP were determined by carotid artery pressure measurement. (D) The expression of miR-1929-3p was detected by reverse transcription-quantitative PCR analysis. (E and F) Effect of MCMV infection on blood vessel wall thickness in mice assessed using hematoxylin and eosin staining (magnification, $x 100$ ). $(\mathrm{G}$ and $\mathrm{H})$ The area of vascular fibrosis was evaluated by Masson's trichrome staining (magnification, $\mathrm{x} 100$ ). " $\mathrm{P}<0.05$ vs. control of the same age; ${ }^{\top} \mathrm{P}<0.05$ vs. MCMV group at 7 months of age. In panels A-C, data were analyzed using two-way ANOVA and Tukey's multiple comparisons post hoc test; in panels D, F and H, data were analyzed using the Student's t-test. n=5 per group. MCMV, murine cytomegalovirus; SBP, systolic blood pressure; DBP, diastolic blood pressure; MAP, mean arterial pressure.

cardiovascular disease $(39,47)$. However, the degree of MCMV infection at 9 months of age was significantly lower compared with that at 12 months of age. This finding was consistent with our previous population survey, which found that an increase in HCMV IgG titer was an independent risk factor for $\mathrm{EH}$ and target organ damage during HCMV infection (48). Another important reason may be that elevated blood pressure is a hemodynamic factor that induces vascular remodeling (49).

A study by Li et al (50) reported that miRNAs may serve as a key link between HCMV and EH. We previously found that CMV infection was associated with EH in Kazakh and Han males in Xinjiang (51). Subsequently, RNA-SEQ screening of miRNAs that were differentially expressed in peripheral blood mononuclear cells from mice with MCMV-induced hypertension revealed that 118 miRNAs were detected between the two groups. Among these, 91 were upregulated and 27 were downregulated, including virus-encoded miRNAs and mouse-encoded miRNAs. This finding indicated that MCMV infection induced differential expression of MCMV and host miRNAs, suggesting that the occurrence and development of MCMV-induced 
A

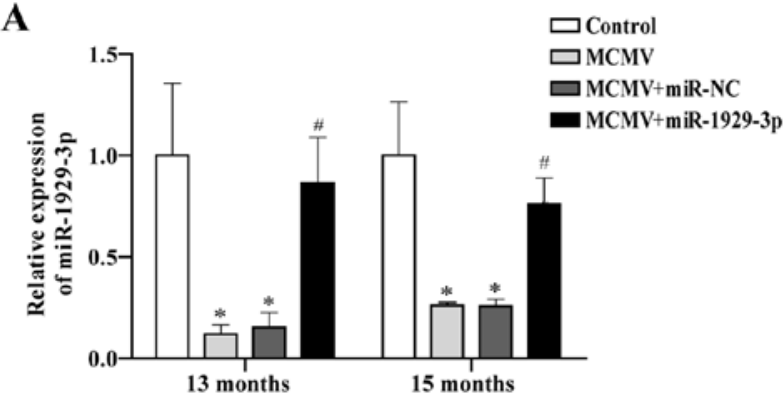

C

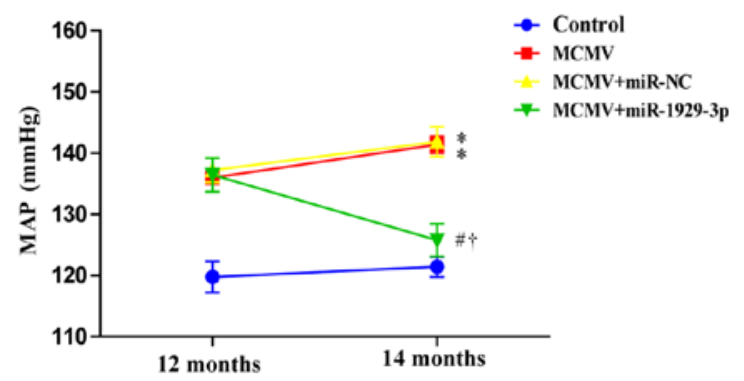

$\mathbf{E}$<smiles>[CH]C1CCCC1</smiles>
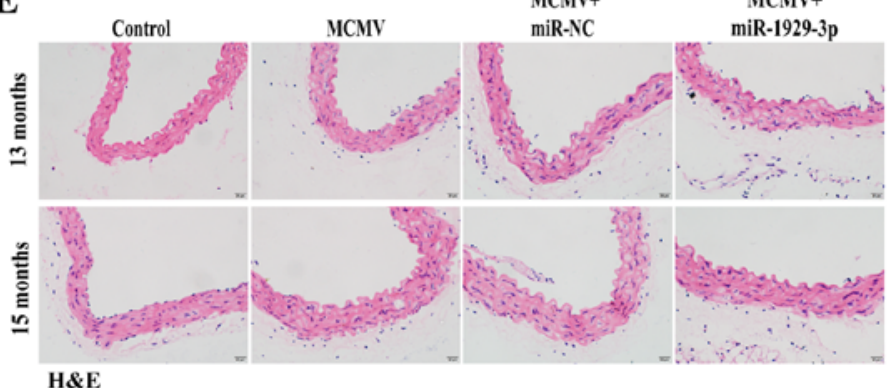

$$
\text { H\&E }
$$

G

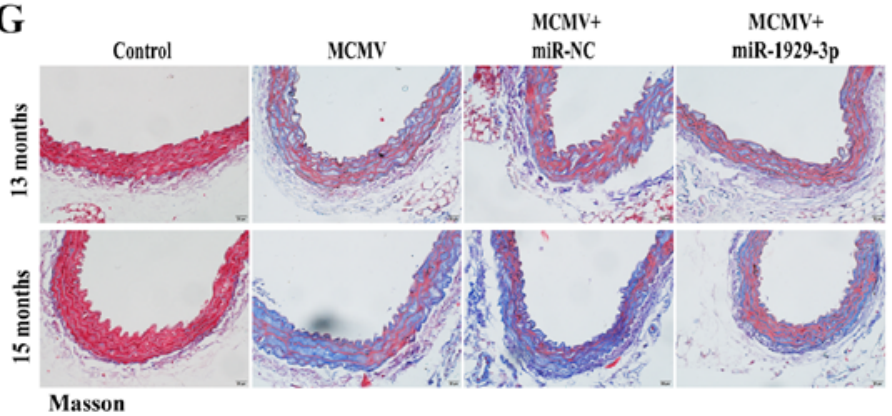

B
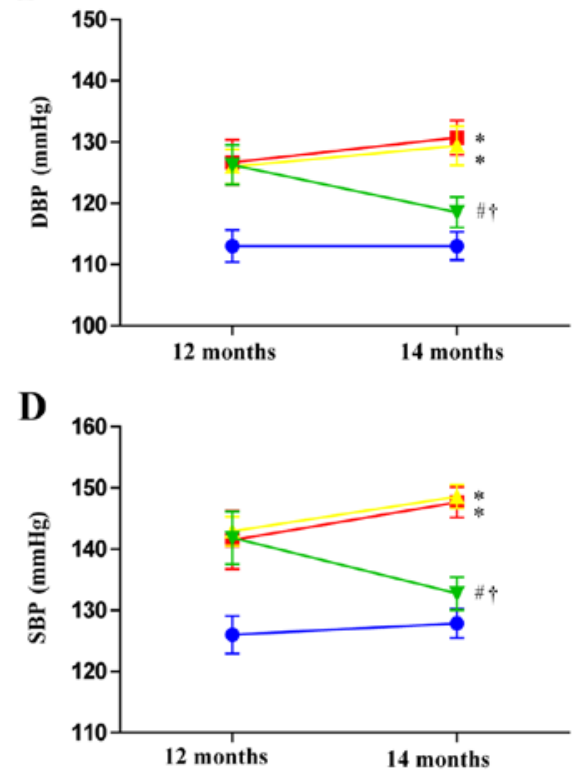

$\mathbf{F}$

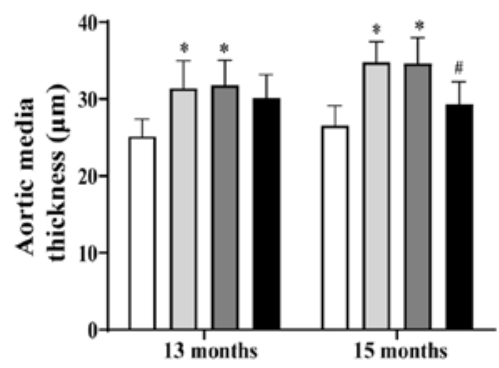

$\mathbf{H}$

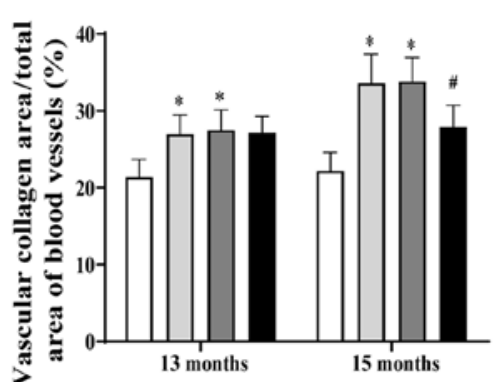

Figure 3. miR-1929-3p overexpression alleviated MCMV-induced vascular remodeling in mice. (A) The expression of miR-1929-3p following transfection with AAV-miR-1929-3p was detected by reverse transcription-quantitative PCR analysis. (B) DBP, (C) MAP and (D) SBP were determined by carotid artery pressure measurement. (E and F) Staining images of aortic tissues following hematoxylin and eosin staining; the pathological changes in aortic tissues were semi-quantitatively evaluated based on histological examination (magnification, $\mathrm{x} 100$ ). ( $\mathrm{G}$ and $\mathrm{H}$ ) The area of vascular fibrosis was evaluated by Masson's trichrome staining (magnification, $\mathrm{x} 100$ ). ${ }^{*} \mathrm{P}<0.05$ vs. control of the same age; ${ }^{\prime} \mathrm{P}<0.05$ vs. MCMV + miR-NC group of the same age; ${ }^{\dagger} \mathrm{P}<0.05$ vs. MCMV group at 7 months of age. In panels A, F and H, data were analyzed using one-way ANOVA, while in panels B-D, data were analyzed using two-way ANOVA, followed by Tukey's multiple comparisons post hoc test; $n=5$ per group. MCMV, murine cytomegalovirus; SBP, systolic blood pressure; DBP, diastolic blood pressure; MAP, mean arterial pressure.

hypertension may involve the interaction of the two miRNAs. Bioinformatics analyses and RT-qPCR validation of peripheral blood monocytes, blood vessels and myocardial tissues revealed that the MMU-miR-1929-3p and MCMV-miR-m01-4-5p results were consistent with those of miRNAs-SEQ sequencing and bioinformatics analysis. However, comparative analysis of the sequence targets of MCMV-miR-m01-4 did not identify any genes associated with hypertension. Therefore, miR-1929-3p was finally selected as the differentially expressed miRNA in MCMV-induced EH. Using bioinformatics analysis and the dual-luciferase reporter assay, Ednra was confirmed as the EH-related target of miR-1929-3p (34). In the present study, RT-qPCR analysis demonstrated that the relative expression level of miR-1929-3p in the MCMV group was significantly decreased at the age of 9 months. However, there was no significant change in the expression of miR-1929-3p at the age of 9-15 months, indicating that the expression of miR-1929-3p did not decrease progressively with the duration of MCMV infection. miRNAs, a class of non-coding single-stranded RNAs with a length of 21-25 nucleotides, are widespread in 
$\mathbf{A}$
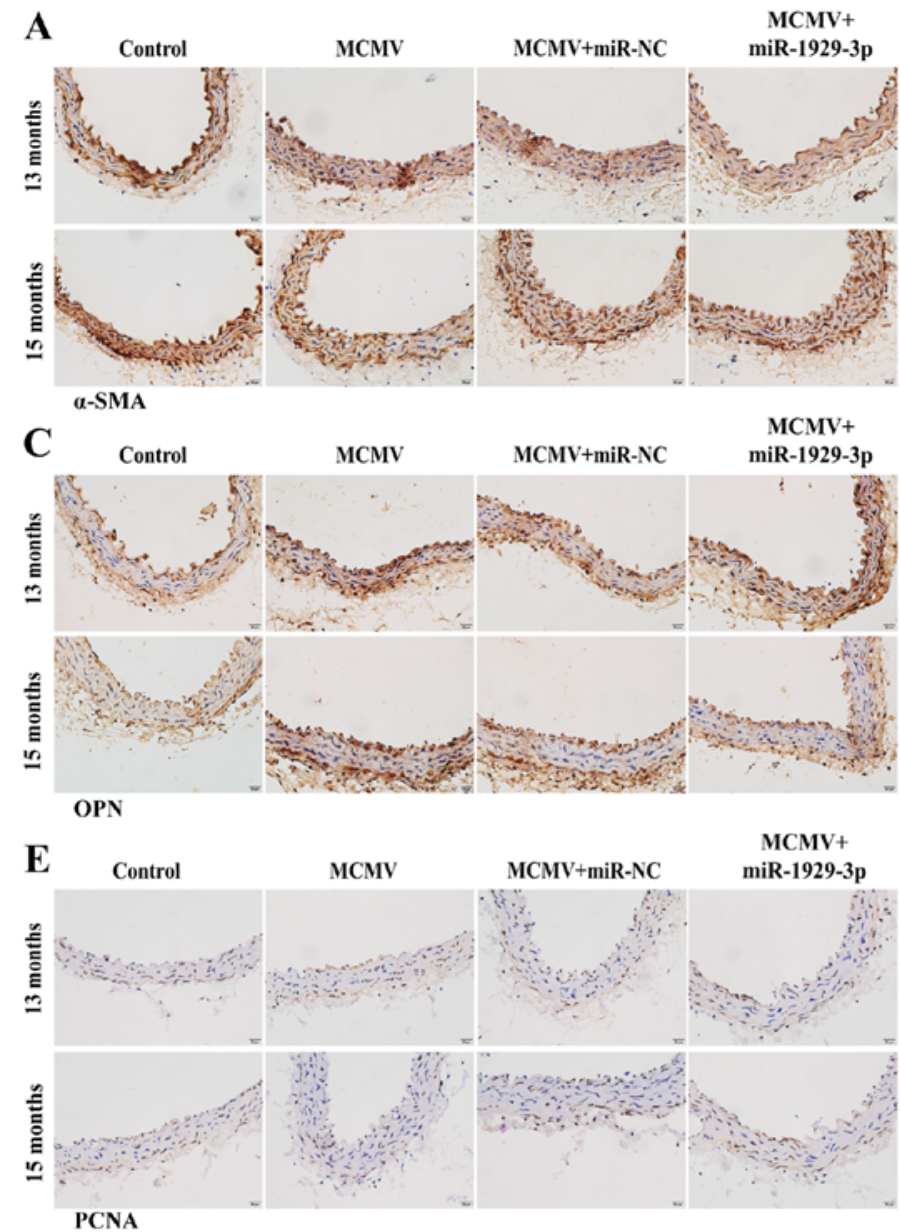

G

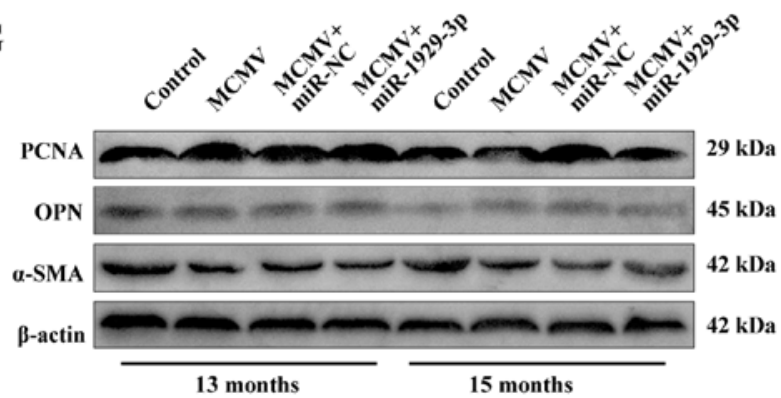

I

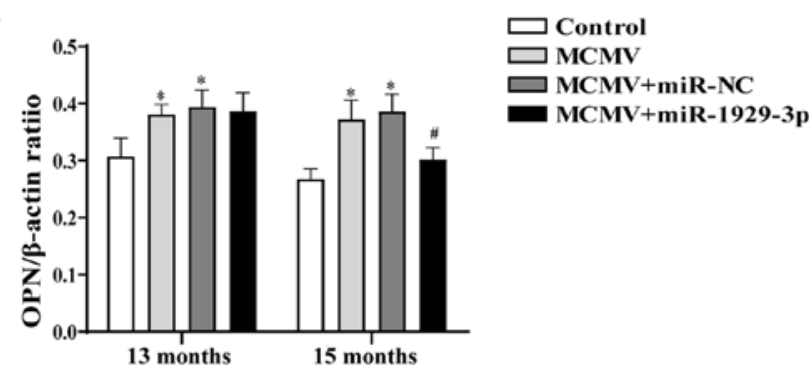

B $\square$ Control

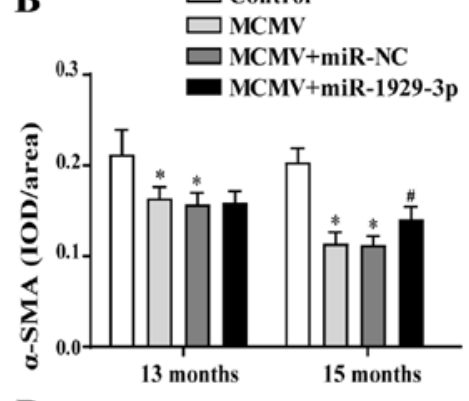

D

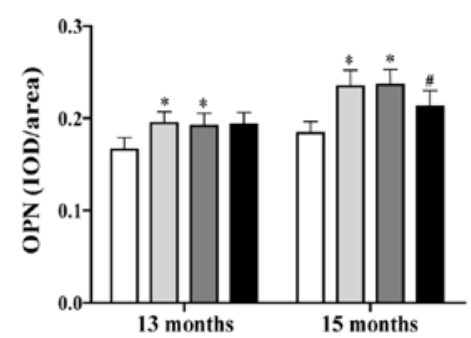

$\mathbf{F}$

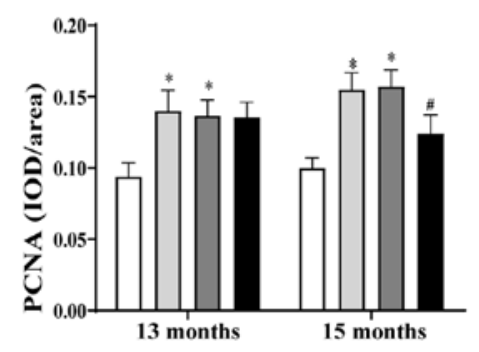

$\mathbf{H}$

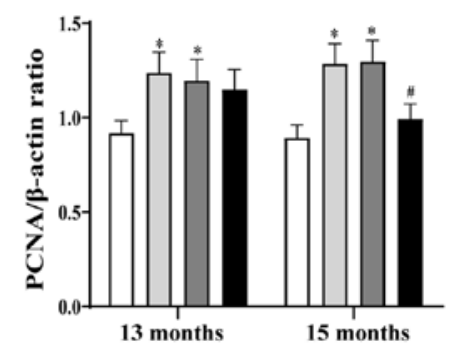

$\mathbf{J}$

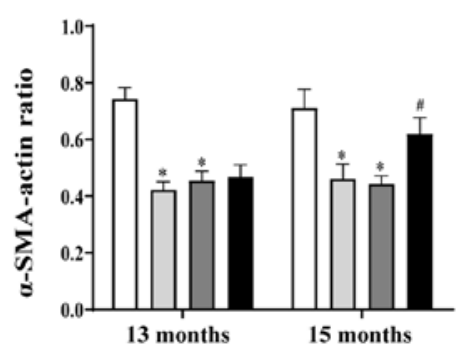

Figure 4. Improved MCMV-induced vascular phenotypic transformation by miR-1929-3p. (A-F) Expression of (A) $\alpha-S M A$, (C) OPN and (E) PCNA in mouse aorta as detected by immunohistochemical staining (magnification, $x 100$ ), and statistical analysis of (B) $\alpha-$ SMA, (D) OPN and (F) PCNA. (G) Western blotting and $(\mathrm{H}-\mathrm{J})$ statistical analysis of the expression of $(\mathrm{H}) \mathrm{PCNA},(\mathrm{I}) \mathrm{OPN}$ and $(\mathrm{J}) \alpha-\mathrm{SMA}$ in aortic tissues. ${ }^{*} \mathrm{P}<0.05$ vs. control of the same age; ${ }^{\prime} \mathrm{P}<0.05$ vs. MCMV $+\mathrm{miR}-\mathrm{NC}$ group of the same age. In panels A, D, F, H, I and J, data were analyzed using one-way ANOVA followed by Tukey's multiple comparisons post hoc. $\mathrm{n}=5$ per group. MCMV, murine cytomegalovirus; SMA, smooth muscle actin; OPN, osteopontin; PCNA, proliferating cell nuclear antigen.

eukaryotes and play important roles in various biological processes, including cell proliferation, differentiation, migration and apoptosis $(52,53)$. Indeed, a large body of evidence indicates that miRNAs are involved in the occurrence and development of EH (54), as well as vascular remodeling (55-57). For example, miR-150 can prevent hypoxia-induced pulmonary vascular remodeling, fibrosis and abnormal proliferation of pulmonary artery smooth muscle cells and endothelial cells (58). These 
EDNRA 3' UTR $\quad$ 5' ...UGUCAAAGUGGCCAAAUGAGCUG...3' IIIIIII mmu-miR-1929-3p 3' GGUGGAUCCAGAGGUACUCGAC 5'

C
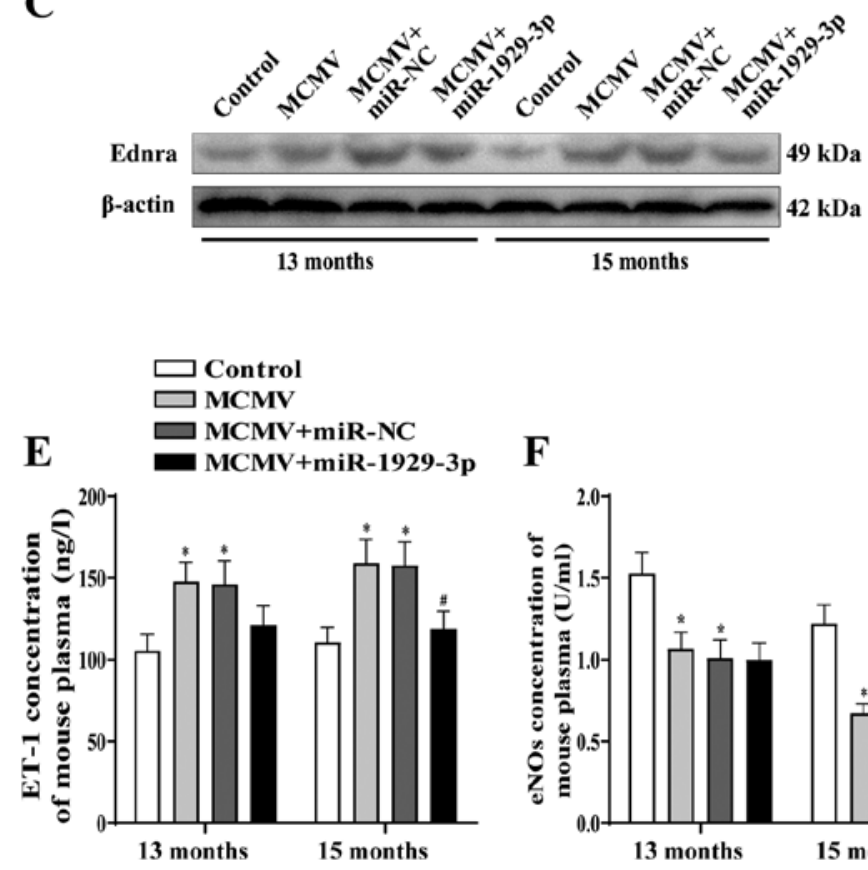

F

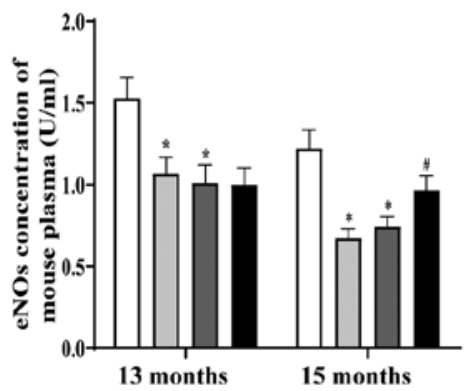

B

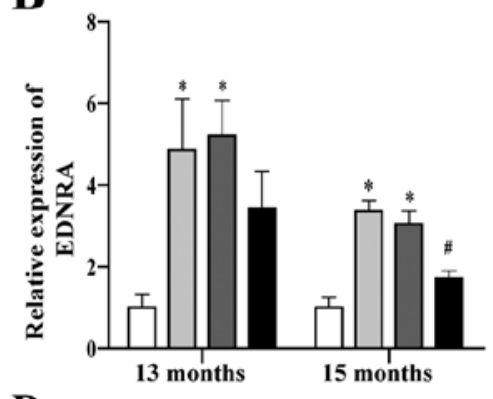

D

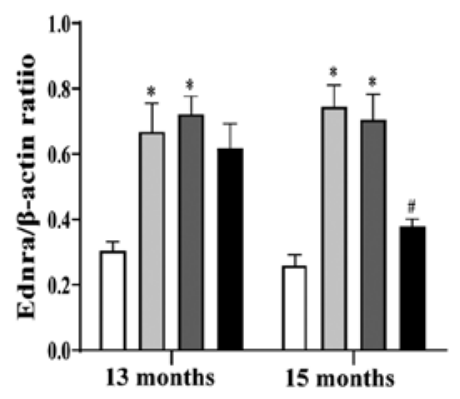

G

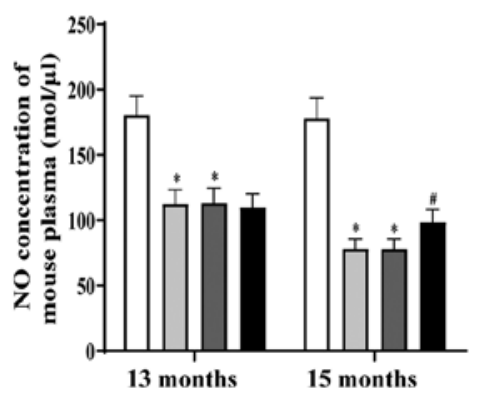

Figure 5. Targeted regulation of Ednra by miR-1929-3p alleviates MCMV-induced endothelial dysfunction. (A) Diagram of miR-1929-3p binding site in Ednra 3' untranslated region. (B) Ednra expression was detected by reverse transcription-quantitative PCR analysis after rAAV-miR-1929-3p transfection in MCMV-infected mice. (C and D) Western blotting and statistical analysis of Ednra following rAAV-miR-1929-3p transfection in MCMV-infected mice. (E) The concentration of ET-1 in the plasma was detected by ELISA. (F) Colorimetry was used to detect eNOS activity in aortic tissue homogenates. (G) Nitrate reduction method was used to detect the concentration of $\mathrm{NO}$ in the homogenate of aortic tissue. ${ }^{*} \mathrm{P}<0.05$ vs. control of the same age; ${ }^{\#} \mathrm{P}<0.05$ vs. $\mathrm{MCMV}+$ miR-NC group of the same age. In panels B, D, E, F and G, data were analyzed using one-way ANOVA followed by Tukey's multiple comparisons post hoc test. $\mathrm{n}=5$ per group. MCMV, murine cytomegalovirus; ET-1, endothelin-1; NO, nitric oxide; eNOS, endothelial nitric oxide synthase.

findings indicate that miRNAs are key regulators of the phenotypic transformation of VSMCs and the adverse remodeling of vessels in hypertension (55). Therefore, it was hypothesized that miR-1929-3p played an important role in MCMV infection of C57BL/6J mice by increasing blood pressure and inducing adverse vascular remodeling.

It was also investigated whether miR-1929-3p played a role in $\mathrm{EH}$ and $\mathrm{MCMV}$-induced vascular remodeling using a recombinant adeno-associated virus-coated miR-1929-3p overexpression plasmid. The data demonstrated that the relative miR-1929-3p expression level in the aortic tissues of C57BL/6J mice was significantly higher compared with that in the MCMV group after 1 month of rAAV-miR-1929-3p intervention, and was even significantly higher after a further 3 months. These results indicated that the targeted vascular delivery of miR-1929-3p mediated by type 9 rAAV significantly increased the relative miR-1929-3p expression level in the aortic tissues of C57BL/6J mice. This finding was consistent with a previous study reporting that type 9 rAAV can be stably transfected into adult mice (59). During the detection of blood pressure in mice, it was observed that the blood pressure in MCMV + miR-1929-3p group mice increased at the age of 14 months, but the vascular function did not improve significantly. However, vascular remodeling was significantly reversed in the MCMV + miR-1929-3p group at 15 months of age. This is consistent with our previous findings, indicating that changes in blood pressure precede vascular remodeling. Furthermore, it was proven that miR-1929-3p overexpression inhibited MCMV-induced VSMC proliferation and differentiation, and exerted a protective effect on blood vessels.

MicroRNAs are post-transcriptional regulators of gene expression that participate in various developmental and cellular processes by inhibiting the translation of target genes (60). Our group identified Ednra as a direct target of miR-1929-3p, which was also verified in the present study. Ednra was significantly upregulated following MCMV-induced miR-1929-3p downregulation. Mainly located in VSMCs, Ednra is primarily involved in the occurrence and development of vasoconstriction, cardiac hypertrophy, inflammation and cardiovascular diseases (61-63). Ednra is activated by ET-1 and induces the 
A
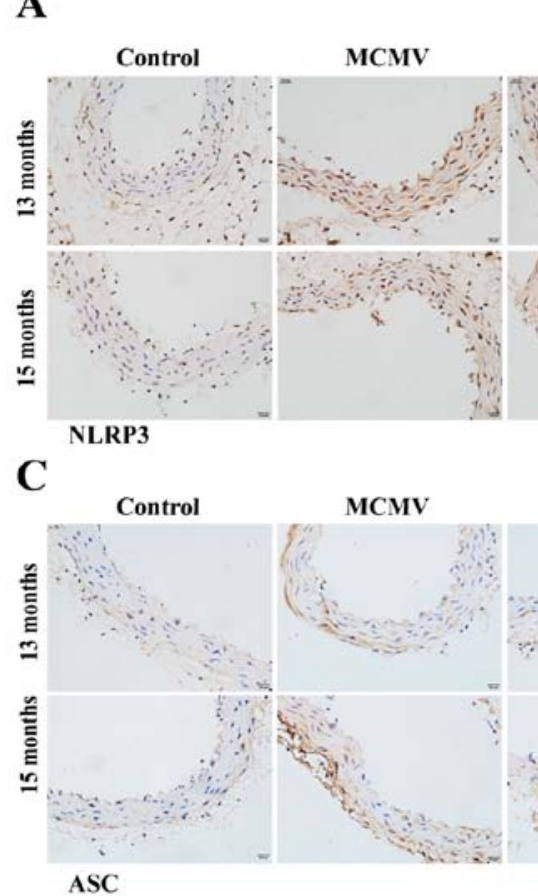

E Control

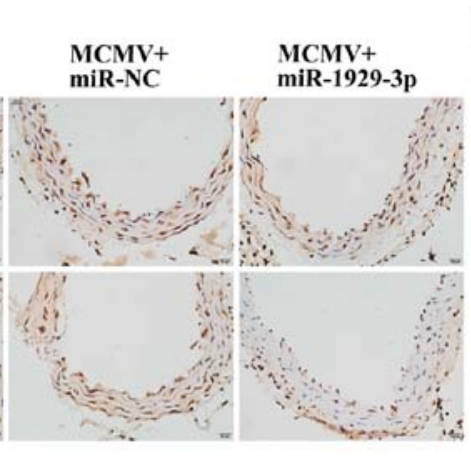

MCMV+
MCMV+ miR-1929-3p

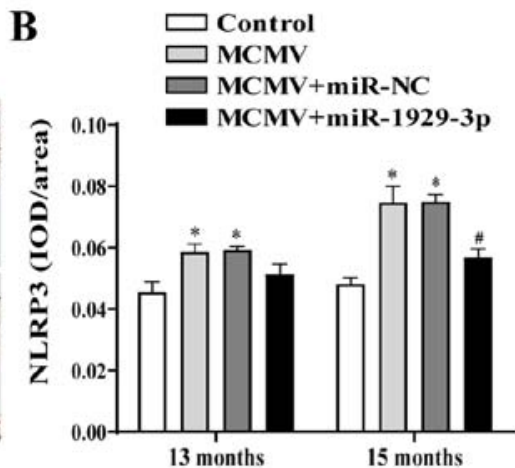

D
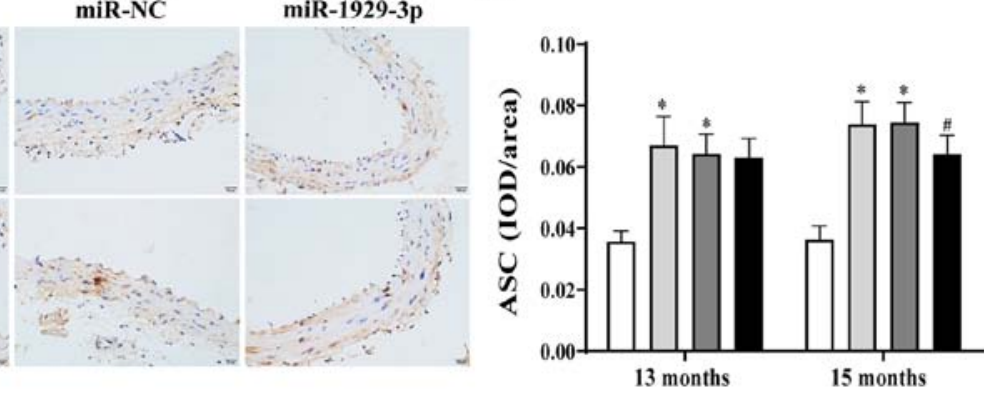

$\mathbf{F}$

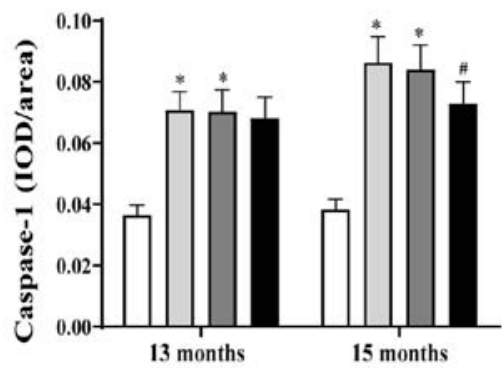

Figure 6. Increased levels of NLRP3, ASC and caspase-1 in the aorta caused by MCMV infection were improved by miR-1929-3p overexpression. (A-F) Immunohistochemical staining and statistical analysis of (A and B) NLRP3, (C and D) ASC and (E and F) caspase-1 in aortic tissues of mice after MCMV infection and miR-1929-3p overexpression (magnification, $\mathrm{x} 100$ ). ${ }^{*} \mathrm{P}<0.05$ vs. control of the same age; ${ }^{\#} \mathrm{P}<0.05$ vs. MCMV + miR-NC group of the same age. In panels $\mathrm{B}, \mathrm{D}$ and F, data were analyzed using one-way ANOVA followed by Tukey's multiple comparisons post hoc test. $n=5$ per group. MCMV, murine cytomegalovirus; NLRP3, nucleotide-binding oligomerization domain-like receptor pyrin domain-containing 3; ASC, apoptosis-associated speck-like protein containing a CARD.

increase of $\mathrm{O}^{2-}$, promotes oxidative stress and causes endothelial cell injury $(64,65)$. Ednra inhibitors can downregulate iNOS to alleviate endotoxin-induced liver injury in cirrhotic patients (61). The present study demonstrated that the downregulation of miR-1929-3p by MCMV increased Ednra levels, thereby leading to increased expression of the endothelial injury factor ET-1 and reduced eNOS activity and NO levels. Other studies have also demonstrated that the activation of Ednra enhances $\mathrm{NF}-\kappa \mathrm{B}$ activation, oxidative stress and production of pro-inflammatory cytokines $(35,66)$. Additionally, TLR-induced NF- $\kappa \mathrm{B}$ activation has been found to lead to the activation of NLRP3, pro-IL-1 and pro-IL-18 (28). However, the association between Ednra and NLRP3 in hypertension has not been widely studied. Therefore, the expression of NLRP3 inflammasome components and downstream IL-1 $\beta$ and IL-18 was examined, and it was observed that IL-1 $\beta$ was significantly inhibited when miR-1929-3p was overexpressed. However, there were no significant effects of miR-1929-3p on IL-18 expression and concentration in the plasma, possibly because the activation of NLRP3 by different cofactors induced different levels of IL-18 and IL-1 $\beta$ processing and secretion (67). Furthermore, Pirhonen et al reported in their studies on the influenza A and Sendai viruses that monocytes and macrophages have different capacities to produce IL-1 $\beta$ and IL-18 due to their different sensitivities to viral infection. In that study, the authors found that the expression of IL-18 was induced only by the Sendai virus, while both the influenza A and Sendai viruses induced IL-1 $\beta$ expression (68), an observation which may partly explain our results. Therefore, it may be inferred that miR-1929-3p inhibits the expression of Ednra, downregulates the activation of the NLRP3 inflammasome and decreases the production of IL-1 $\beta$ in MCMV-infected hypertensive mice. A limitation of the present study was that the mechanism underlying the involvement of Ednra/NLRP3 in the development of hypertension and vascular remodeling following MCMV-induced downregulation of miR-1929-3p in mice was not fully elucidated. Future work will aim to study these mechanisms in further detail at the cellular and molecular level. Furthermore, it is well known that miRNAs have a complex regulatory network, which can either regulate the expression of multiple genes through a 

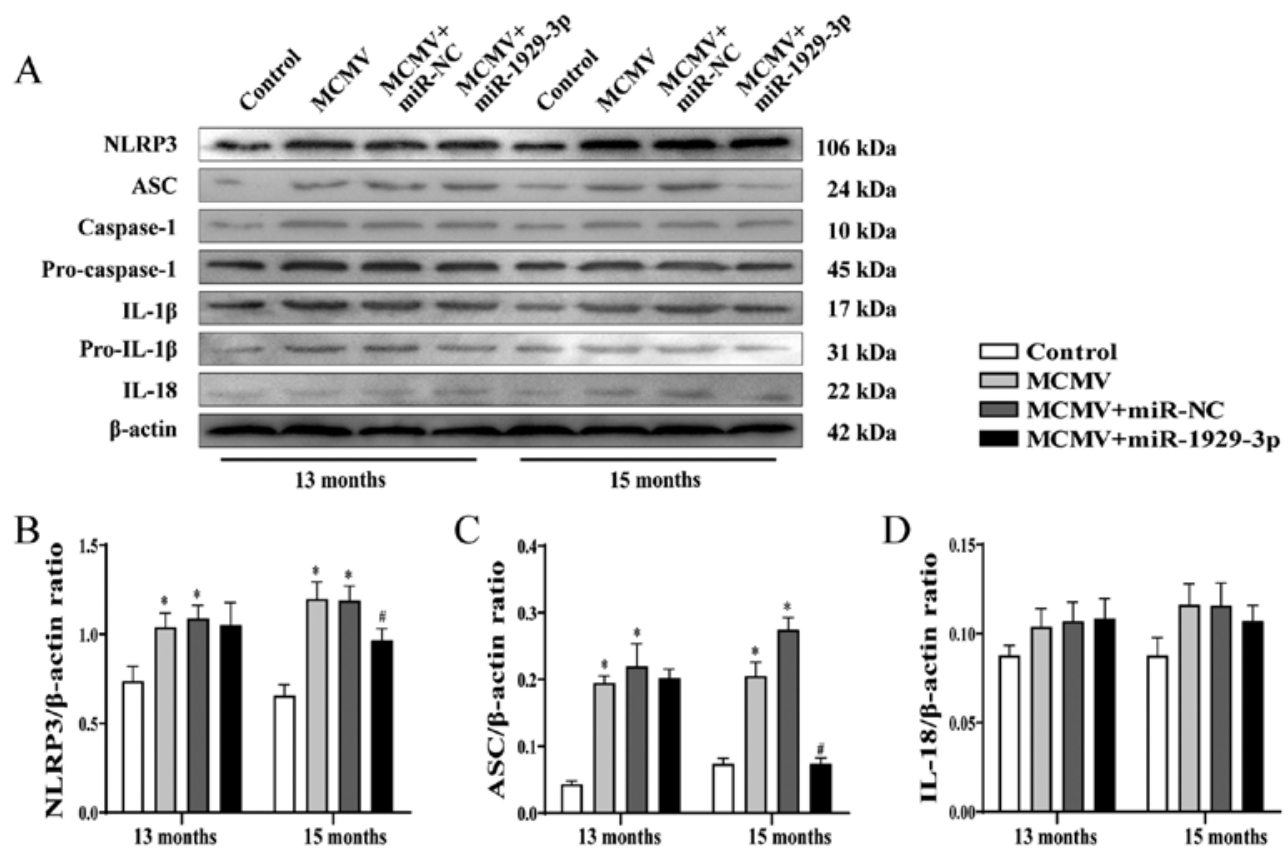

$\mathrm{E}$

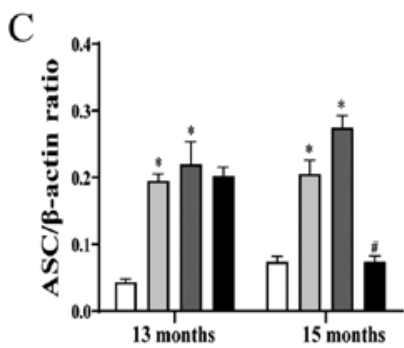

$\mathrm{D}$

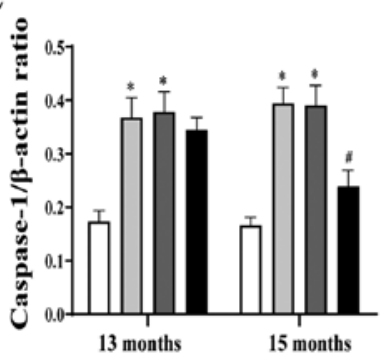

F
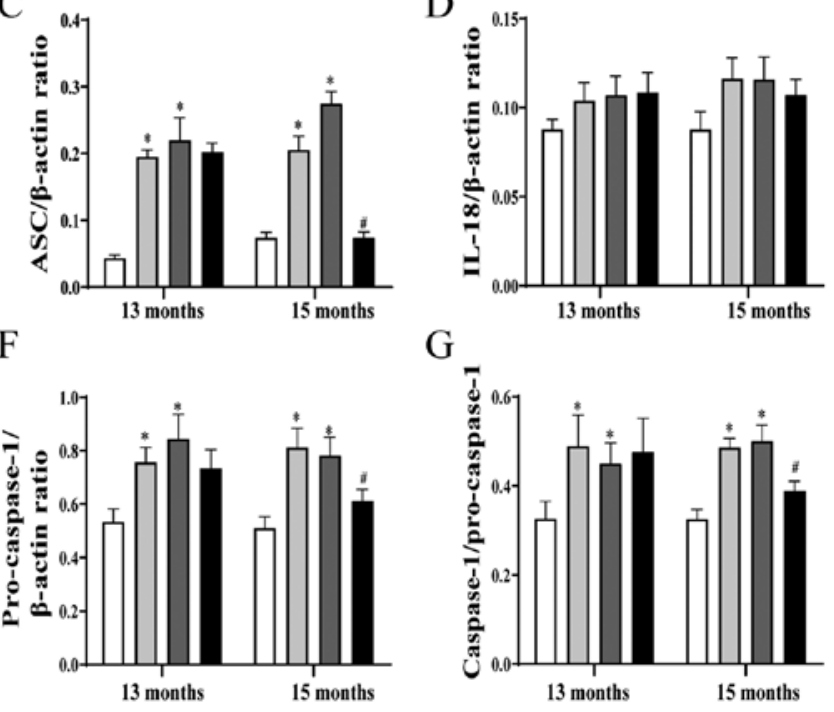

G
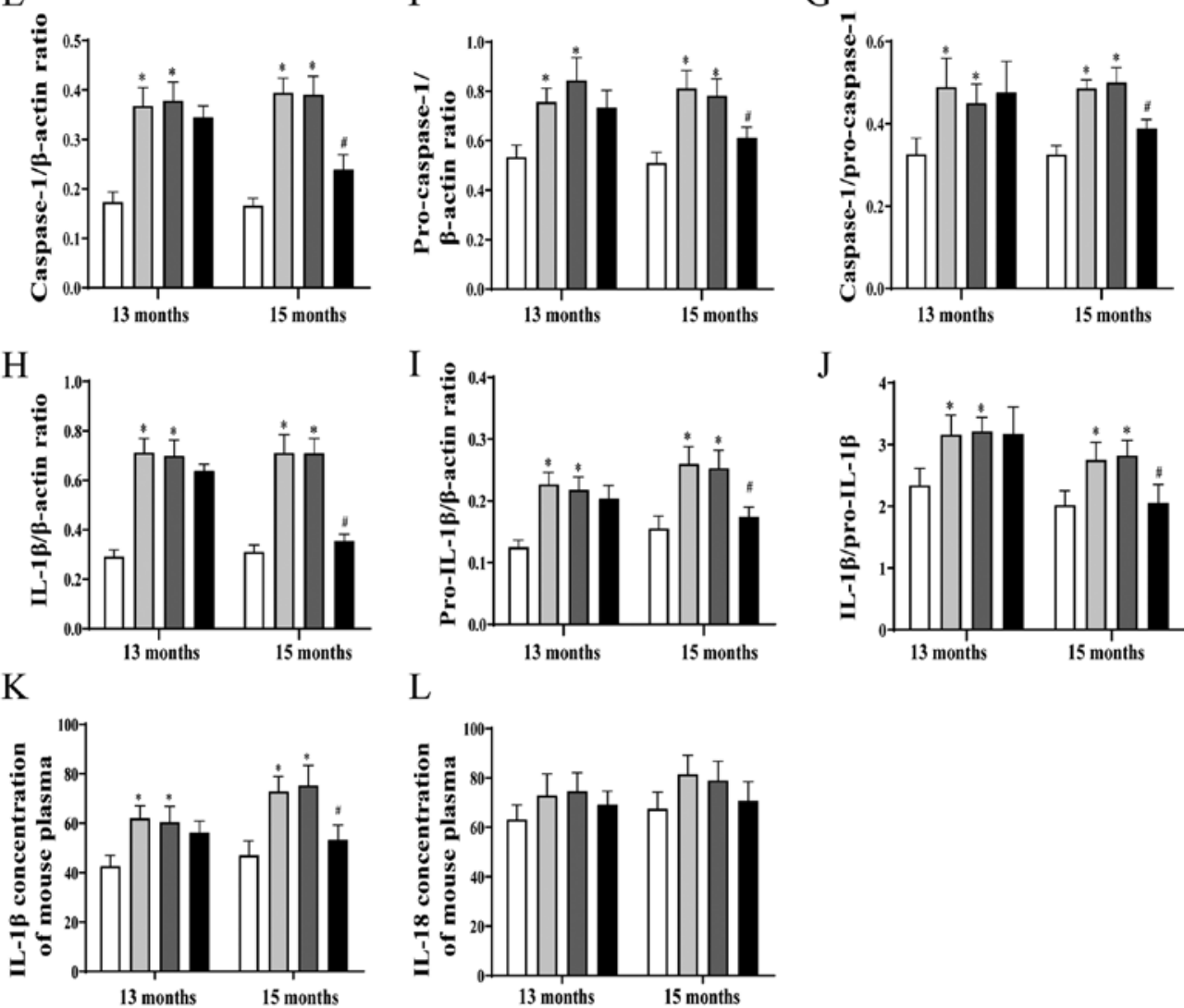

$\mathrm{L}$
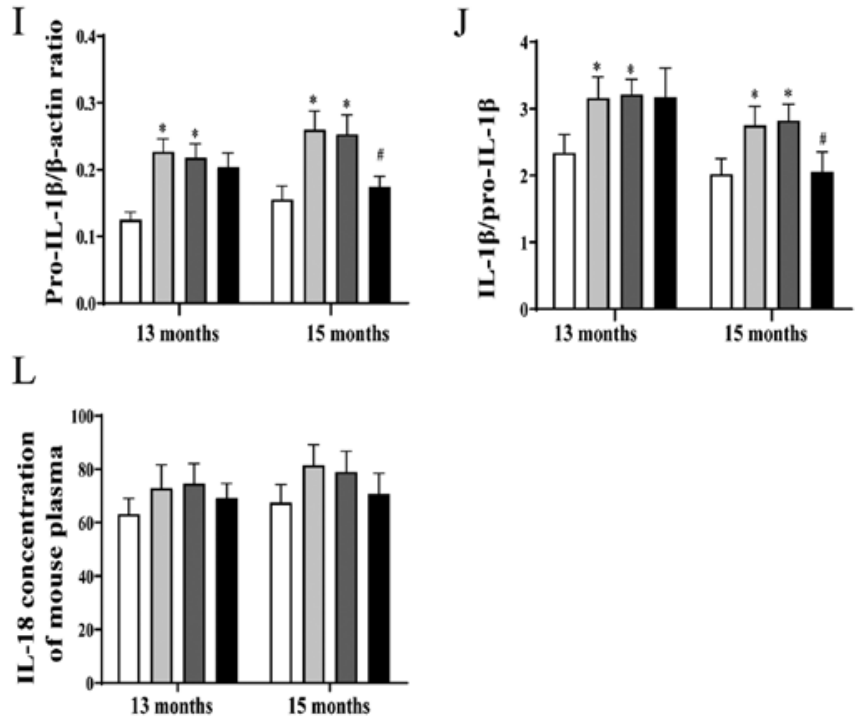

Figure 7. Overexpression of miR-1929-3p alleviates MCMV-induced NLRP3 inflammasome activation. (A) Western blotting and (B-L) statistical analysis of the expression of inflammatory factors in aortic tissues of mice after MCMV infection and miR-1929-3p overexpression. (B) NLRP3, (C) ASC, (D) IL-18, (E) caspase-1, (F) pro-caspase-1, (G) caspase-1/pro-caspase-1 ratio; (H) IL-1 $\beta$, (I) pro-IL-1 $\beta$, (J) IL-1 $\beta /$ pro-IL-1 $\beta$ ratio and (K) IL-1 $\beta$ in mouse plasma. ${ }^{*} \mathrm{P}<0.05$ vs. control of the same age; ${ }^{~} \mathrm{P}<0.05$ vs. MCMV + miR-NC group of the same age. In panels B-L, data were analyzed using one-way ANOVA followed by Tukey's multiple comparisons post hoc test. $\mathrm{n}=5$ per group. MCMV, murine cytomegalovirus; NLRP3, nucleotide-binding oligomerization domain-like receptor pyrin domain-containing 3; ASC, apoptosis-associated speck-like protein containing a CARD; IL-1 $\beta$, interleukin 1 $\beta$; IL-18, interleukin 18.

single miRNA, or regulate the expression of a single gene via multiple miRNAs (69). Although miR-1929-3p was found to improve MCMV-induced hypertensive vascular remodeling by inhibiting Ednra, whether other targets of miR-1929-3p may be partially involved in promoting the miR-1929-3p-mediated proliferation of blood vessels cannot be ruled out. Moreover, the possibility that MCMV-induced differential expression of other miRNAs and proteins may affect blood pressure and vascular remodeling in mice must be considered. Furthermore, since miR-1929-3p is a murine miRNA, it cannot be confirmed 
whether it was specifically differentially expressed only in response to MCMV infection.

In summary, the present study demonstrated that MCMV induced an increase in blood pressure and promoted vascular remodeling in mice, which was likely caused by the downregulation of miR-1929-3p and the activation of ET-1/Ednra/NLRP3 inflammasome. Overexpression of miR-1929-3p alleviated adverse MCMV-induced vascular remodeling via a mechanism likely involving the inhibition of ET-1/Ednra and subsequent deactivation of the NLRP3 inflammasome. Since miRNAs from different species are highly homologous, miR-1929-3p may represent a novel CMV-mediated hypertension biomarker and a potential target for regulating vascular remodeling.

\section{Acknowledgements}

Not applicable.

\section{Funding}

The present study was supported by grants from the National Natural Science Foundation of China (no. 31760291), the National College Students innovation and Entrepreneurship Training Program (no. 202010759027), and the Scientific Research Project of Shihezi University (no. ZZZC201921A).

\section{Availability of data and materials}

All data generated or analyzed during the present study are included in this published article or are available from the corresponding author on reasonable request.

\section{Authors' contributions}

YS, FH and DX conceived and designed the experiments. WZ, YS, DX, ZH, ZZ and YW performed the experiments. NT, NL, LW, JP and YT analyzed the data. WZ, YL, HZ and FH wrote or modified the manuscript. YS, ZH and FH confirm the authenticity of all the raw data. All the authors have read and approved the final manuscript.

\section{Ethics approval and consent to participate}

All animal studies were performed according to the guidelines of the Chinese Council on Animal Care, and the study was approved by the Ethics Committee of Shihezi Medical University (Shihezi, China).

\section{Patient consent for publication}

Not applicable.

\section{Competing interests}

The authors declare that they have no competing interests.

\section{References}

1. Renna NF, de Las Heras N and Miatello RM: Pathophysiology of vascular remodeling in hypertension. Int J Hypertens 2013: 808353, 2013.
2. Kearney PM, Whelton M, Reynolds K, Muntner P, Whelton PK and He J: Global burden of hypertension: Analysis of worldwide data. Lancet 365: 217-223, 2005.

3. Chiong JR: Controlling hypertension from a public health perspective. Int J Cardiol 127: 151-156, 2008.

4. Bloch MJ: Worldwide prevalence of hypertension exceeds 1.3 billion. J Am Soc Hypertens 10: 753-754, 2016.

5. Sun F, Zhang K, Li X, He N, Zhao J and Qiu C: Effects of angiotensin-converting enzyme gene and environment interaction on essential hypertension in the Han nationality. Wei Sheng Yan Jiu 46: 378-383, 2017 (In Chinese).

6. Shah SFA, Iqbal T, Qamar R, Rafiq MA and Hussain S: ARG1 gene polymorphisms and their association in individuals with essential hypertension: A case-control study. DNA Cell Biol 37: 609-616, 2018.

7. Li C, Samaranayake NR, Ong KL, Wong HK and Cheung BM: Is human cytomegalovirus infection associated with hypertension? The United States National Health and Nutrition Examination Survey 1999-2002. PLoS One 7: e39760, 2012.

8. Sun HJ, Ren XS, Xiong XQ, Chen YZ, Zhao MX, Wang JJ, Zhou YB, Han Y, Chen Q, Li YH, et al: NLRP3 inflammasome activation contributes to VSMC phenotypic transformation and proliferation in hypertension. Cell Death Dis 8: e3074, 2017.

9. Hijmans JG, Diehl KJ, Bammert TD, Kavlich PJ, Lincenberg GM, Greiner JJ, Stauffer BL and DeSouza CA: Association between hypertension and circulating vascular-related microRNAs. J Hum Hypertens 32: 440-447, 2018.

10. Murphy E, Yu D, Grimwood J, Schmutz J, Dickson M, Jarvis MA, Hahn G, Nelson JA, Myers RM and Shenk TE: Coding potential of laboratory and clinical strains of human cytomegalovirus. Proc Natl Acad Sci USA 100: 14976-14981, 2003.

11. Gatherer D, Seirafian S, Cunningham C, Holton M, Dargan DJ, Baluchova K, Hector RD, Galbraith J, Herzyk P, Wilkinson GW and Davison AJ: High-resolution human cytomegalovirus transcriptome. Proc Natl Acad Sci USA 108: 19755-19760, 2011.

12. Cannon MJ, Schmid DS and Hyde TB: Review of cytomegalovirus seroprevalence and demographic characteristics associated with infection. Rev Med Virol 20: 202-213, 2010.

13. Zhang M, Yang Y, Yang X and Cai J: Human cytomegalovirus infection is a novel etiology for essential hypertension. Med Hypotheses 76: 682-684, 2011.

14. Emery VC: Cytomegalovirus: Recent progress in understanding pathogenesis and control. QJM 105: 401-405, 2012.

15. Fu M, Gao Y, Zhou Q, Zhang Q, Peng Y, Tian K, Wang J and Zheng X: Human cytomegalovirus latent infection alters the expression of cellular and viral microRNA. Gene 536: 272-278, 2014.

16. Leite-Moreira AM, Lourenço AP, Falcão-Pires I and Leite-Moreira AF: Pivotal role of microRNAs in cardiac physiology and heart failure. Drug Discov Today 18: 1243-1249, 2013.

17. Lu TX and Rothenberg ME: MicroRNA. J Allergy Clin Immunol 141: 1202-1207, 2018.

18. Li X, Wei Y and Wang Z: microRNA-21 and hypertension. Hypertens Res 41: 649-661, 2018.

19. Huo KG, Richer C, Berillo O, Mahjoub N, Fraulob-Aquino JC, Barhoumi T, Ouerd S, Coelho SC, Sinnett D, Paradis P and Schiffrin EL: miR-431-5p knockdown protects against angiotensin II-induced hypertension and vascular injury. Hypertension 73: 1007-1017, 2019.

20. Li FJ, Zhang CL, Luo XJ, Peng J and Yang TL: Involvement of the MiR-181b-5p/HMGB1 pathway in Ang II-induced phenotypic transformation of smooth muscle cells in hypertension. Aging Dis 10: 231-248, 2019.

21. Shen K, Xu L, Chen D, Tang W and Huang Y: Human cytomegalovirus-encoded miR-UL112 contributes to HCMV-mediated vascular diseases by inducing vascular endothelial cell dysfunction. Virus Genes 54: 172-181, 2018.

22. Zhang S, Liu L, Wang R, Tuo H, Guo Y, Yi L, Wang D and Wang J: miR-138 promotes migration and tube formation of human cytomegalovirus-infected endothelial cells through the SIRT1/p-STAT3 pathway. Arch Virol 162: 2695-2704, 2017.

23. Pfeffer S, Sewer A, Lagos-Quintana M, Sheridan R, Sander C, Grässer FA, van Dyk LF, Ho CK, Shuman S, Chien M, et al: Identification of microRNAs of the herpesvirus family. Nat Methods 2: 269-276, 2005.

24. Meshesha MK, Veksler-Lublinsky I, Isakov O, Reichenstein I, Shomron N, Kedem K, Ziv-Ukelson M, Bentwich Z and Avni YS: The microRNA transcriptome of human cytomegalovirus (HCMV). Open Virol J 6: 38-48, 2012.

25. Zhang L, Yu J and Liu Z: MicroRNAs expressed by human cytomegalovirus. Virol J 17: 34, 2020. 
26. Elliott EI and Sutterwala FS: Initiation and perpetuation of NLRP3 inflammasome activation and assembly. Immunol Rev 265: 35-52, 2015.

27. Krishnan SM, Sobey CG, Latz E, Mansell A and Drummond GR IL-1 $\beta$ and IL-18: Inflammatory markers or mediators of hypertension? Br J Pharmacol 171: 5589-5602, 2014.

28. Liu D, Zeng X, Li X, Mehta JL and Wang X: Role of NLRP3 inflammasome in the pathogenesis of cardiovascular diseases. Basic Res Cardiol 113: 5, 2018

29. Intengan HD and Schiffrin EL: Vascular remodeling in hypertension: Roles of apoptosis, inflammation, and fibrosis. Hypertension 38: 581-587, 2001.

30. Song J, Yang S, Yin R, Xiao Q, Ma A and Pan X: MicroRNA-181a regulates the activation of the NLRP3 inflammatory pathway by targeting MEK1 in THP-1 macrophages stimulated by ox-LDL. J Cell Biochem 120: 13640-13650, 2019.

31. Wang J, Wu Q, Yu J, Cao X and Xu Z: miR-125a-5p inhibits the expression of NLRP3 by targeting CCL4 in human vascular smooth muscle cells treated with ox-LDL. Exp Ther Med 18 $1645-1652,2019$.

32. Ren XS, Tong Y, Ling L, Chen D, Sun HJ, Zhou H, Qi XH, Chen Q, Li YH, Kang YM and Zhu GQ: NLRP3 gene deletion attenuates Angiotensin II-induced phenotypic transformation of vascular smooth muscle cells and vascular remodeling. Cell Physiol Biochem 44: 2269-2280, 2017.

33. Wang R, Wu W, Li W, Huang S, Li Z, Liu R, Shan Z, Zhang C, $\mathrm{Li}$ W and Wang S: Activation of NLRP3 inflammasome promotes foam cell formation in vascular smooth muscle cells and atherogenesis via HMGB1. J Am Heart Assoc 7: e008596, 2018

34. Yunzhong S, Xi D, Zhang X, Zhen H, Tang N, YongMin L, Wang LM, Tang Y, Zhong H and He F: Screening and validation of differentially expressed microRNAs and target genes in hypertensive mice induced by cytomegalovirus infection. Biosci Rep: BSR20202387, 2020.

35. Yeager ME, Belchenko DD, Nguyen CM, Colvin KL, Ivy DD and Stenmark KR: Endothelin-1, the unfolded protein response, and persistent inflammation: Role of pulmonary artery smooth muscle cells. Am J Respir Cell Mol Biol 46: 14-22, 2012.

36. Livak KJ and Schmittgen TD: Analysis of relative gene expression data using real-time quantitative PCR and the 2(-Delta Delta C(T)) method. Methods 25: 402-408, 2001.

37. Shiao YH, Palli D, Caporaso NE, Alvord WG, Amorosi A, Nesi G, Saieva C, Masala G, Fraumeni JF Jr and Rice JM Genetic and immunohistochemical analyses of p53 independently predict regional metastasis of gastric cancers. Cance Epidemiol Biomarkers Prev 9: 631-633, 2000.

38. Touyz RM and Schiffrin EL: Role of endothelin in human hypertension. Can J Physiol Pharmacol 81: 533-541, 2003.

39. Roberts ET, Haan MN, Dowd JB and Aiello AE: Cytomegalovirus antibody levels, inflammation, and mortality among elderly Latinos over 9 years of follow-up. Am J Epidemiol 172: 363-371, 2010.

40. Ward R and Ergul A: Relationship of endothelin-1 and NLRP3 inflammasome activation in HT22 hippocampal cells in diabetes Life Sci 159: 97-103, 2016

41. Reddehase MJ and Lemmermann NAW: Cellular reservoirs of latent cytomegaloviruses. Med Microbiol Immunol 208 : 391-403, 2019.

42. Cheng J, Ke Q, Jin Z, Wang H, Kocher O, Morgan JP, Zhang J and Crumpacker CS: Cytomegalovirus infection causes an increase of arterial blood pressure. PLoS Pathog 5: e1000427, 2009.

43. Bruno RM, Duranti E, Ippolito C, Segnani C, Bernardini N, Di Candio G, Chiarugi M, Taddei S and Virdis A: Different impact of essential hypertension on structural and functional age-related vascular changes. Hypertension 69: 71-78, 2017.

44. Dinh QN, Drummond GR, Kemp-Harper BK, Diep H, De Silva TM, Kim HA, Vinh A, Robertson AAB, Cooper MA, Mansell A, et al: Pressor response to angiotensin II is enhanced in aged mice and associated with inflammation, vasoconstriction and oxidative stress. Aging (Albany NY) 9: 1595-1606, 2017.

45. Baumbach GL and Ghoneim S: Vascular remodeling in hypertension. Scanning Microsc 7: 137-143, 1993.

46. Laurent S and Boutouyrie P: The structural factor of hypertension: Large and small artery alterations. Circ Res 116: 1007-1021, 2015.

47. Wang GC, Kao WH, Murakami P, Xue QL, Chiou RB, Detrick B, McDyer JF, Semba RD, Casolaro V, Walston JD and Fried LP: Cytomegalovirus infection and the risk of mortality and frailty in older women: A prospective observational cohort study. Am J Epidemiol 171: 1144-1152, 2010.
48. Li Z, Tang Y, Tang N, Feng Q, Zhong H, Liu YM, Wang LM and He F: High anti-human cytomegalovirus antibody levels are associated with the progression of essential hypertension and target organ damage in Han Chinese population. PLoS One 12: e0181440, 2017.

49. Mulvany MJ: Small artery remodeling and significance in the development of hypertension. News Physiol Sci 17: 105-109, 2002.

50. Li S, Zhu J, Zhang W, Chen Y, Zhang K, Popescu LM, Ma X, Lau WB, Rong R, Yu X, et al: Signature microRNA expression profile of essential hypertension and its novel link to human cytomegalovirus infection. Circulation 124: 175-184, 2011.

51. Tang N, Li JW, Liu YM, Zhong H, Wang LM, Deng FM, Qu YY, Hui J, Cheng J, Tang B, et al: Human cytomegalovirus infection is associated with essential hypertension in Kazakh and Han Chinese populations. Med Sci Monit 20: 2508-2519, 2014.

52. Bronze-da-Rocha E: MicroRNAs expression profiles in cardiovascular diseases. Biomed Res Int 2014: 985408, 2014.

53. Song X, Shan D, Chen J and Jing Q: miRNAs and lncRNAs in vascular injury and remodeling. Sci China Life Sci 57: 826-835, 2014.

54. Jeppesen PL, Christensen GL, Schneider M, Nossent AY, Jensen HB, Andersen DC, Eskildsen T, Gammeltoft S, Hansen JL and Sheikh SP: Angiotensin II type 1 receptor signalling regulates microRNA differentially in cardiac fibroblasts and myocytes. Br J Pharmacol 164: 394-404, 2011.

55. Xie C, Zhang J and Chen YE: Chapter Fifteen-MicroRNA and Vascular Smooth Muscle Cells. Elsevier Science \& Technology, 2011.

56. Kang $\mathrm{H}$ and Hata A: MicroRNA regulation of smooth muscle gene expression and phenotype. Curr Opin Hematol 19: 224-231, 2012.

57. Robinson $\mathrm{HC}$ and Baker AH: How do microRNAs affect vascular smooth muscle cell biology? Curr Opin Lipidol 23: 405-411, 2012

58. Li Y, Ren W, Wang X, Yu X, Cui L, Li X, Zhang X and Shi B: MicroRNA-150 relieves vascular remodeling and fibrosis in hypoxia-induced pulmonary hypertension. Biomed Pharmacother 109: 1740-1749, 2019.

59. Bostick B, Ghosh A, Yue Y, Long C and Duan D: Systemic AAV-9 transduction in mice is influenced by animal age but not by the route of administration. Gene Ther 14: 1605-1609, 2007.

60. Krol J, Loedige I and Filipowicz W: The widespread regulation of microRNA biogenesis, function and decay. Nat Rev Genet 11: 597-610, 2010.

61. Keller S, Karaa A, Paxian M, Clemens MG and Zhang JX: Inhibition of endothelin-1-mediated up-regulation of iNOS by bosentan ameliorates endotoxin-induced liver injury in cirrhosis. Shock 25: 306-313, 2006.

62. Khalil RA: Modulators of the vascular endothelin receptor in blood pressure regulation and hypertension. Curr Mol Pharmacol 4: 176-186, 2011.

63. D'Orléans-Juste P, Akide Ndunge OB, Desbiens L, Tanowitz HB and Desruisseaux MS: Endothelins in inflammatory neurological diseases. Pharmacol Ther 194: 145-160, 2019.

64. Xu H, Lin L and Yuan WJ: Antiarrhythmic effect of endothelin-A receptor antagonist on acute ischemic arrhythmia in isolated rat heart. Acta Pharmacol Sin 24: 37-44, 2003.

65. Elmarakby AA, Loomis ED, Pollock JS and Pollock DM: NADPH oxidase inhibition attenuates oxidative stress but not hypertension produced by chronic ET-1. Hypertension 45: 283-287, 2005 .

66. Loomis ED, Sullivan JC, Osmond DA, Pollock DM and Pollock JS: Endothelin mediates superoxide production and vasoconstriction through activation of NADPH oxidase and uncoupled nitric-oxide synthase in the rat aorta. J Pharmacol Exp Ther 315: 1058-1064, 2005.

67. Schmidt RL and Lenz LL: Distinct licensing of IL-18 and IL-1 secretion in response to NLRP3 inflammasome activation. PLoS One 7: e45186, 2012

68. Pirhonen J, Sareneva T, Kurimoto M, Julkunen I and Matikainen S: Virus infection activates IL-1 beta and IL-18 production in human macrophages by a caspase-1-dependent pathway. J Immunol 162: 7322-7329, 1999.

69. Mohr AM and Mott JL: Overview of microRNA biology. Semin Liver Dis 35: 3-11, 2015.

This work is licensed under a Creative Commons Attribution-NonCommercial-NoDerivatives 4.0 International (CC BY-NC-ND 4.0) License. 